\title{
Numerical, Approximate Solutions, and Optimal Control on the Deathly Lassa Hemorrhagic Fever Disease in Pregnant Women
}

\author{
M. Higazy $\mathbb{D}^{1,2}$ A. El-Mesady $\mathbb{D}^{2},{ }^{2}$ A. M. S. Mahdy $\mathbb{D}^{1,3}$ Sami Ullah, $^{4,5}$ and A. Al-Ghamdi ${ }^{6}$ \\ ${ }^{1}$ Department of Mathematics and Statistics, College of Science, Taif University, P.O. Box 11099, Taif 21944, Saudi Arabia \\ ${ }^{2}$ Department of Physics and Engineering Mathematics, Faculty of Electronic Engineering, Menoufia University, \\ Menouf 32952, Egypt \\ ${ }^{3}$ Department of Mathematics, Faculty of Science, Zagazig University, Zagazig, Egypt \\ ${ }^{4}$ Research Center for Advanced Materials Science (RCAMS), King Khalid University, P.O. Box 9004, Abha 61413, Saudi Arabia \\ ${ }^{5}$ Department of Chemistry, College of Science, King Khalid University, P.O. Box 9004, Abha 61413, Saudi Arabia \\ ${ }^{6}$ Department of Physics, Faculty of Science, King Abdulaziz University, Jeddah 21589, Saudi Arabia
}

Correspondence should be addressed to A. El-Mesady; ahmed.ibrahiem81@el-eng.menofia.edu.eg

Received 26 October 2021; Revised 13 November 2021; Accepted 22 November 2021; Published 15 December 2021

Academic Editor: Youssri Hassan Youssri

Copyright (c) 2021 M. Higazy et al. This is an open access article distributed under the Creative Commons Attribution License, which permits unrestricted use, distribution, and reproduction in any medium, provided the original work is properly cited.

\begin{abstract}
This paper is devoted to the model of Lassa hemorrhagic fever (LHF) disease in pregnant women. This disease is a biocidal fever and epidemic. LHF disease in pregnant women has negative impacts that were initially appeared in Africa. In the present study, we find an approximate solution to the fractional-order model that describes the fatal LHF disease. Laplace transforms coupled with the Adomian decomposition method (ADM) are applied. In addition, the fractional-order LHF model is numerically simulated in terms of a varied fractional order. Furthermore, a fractional order optimal control for the LHF model is studied.
\end{abstract}

\section{Introduction}

Infectious diseases remain a major threat to human health and welfare throughout history. Their spread is affected by several factors such as mode of transmission, infectious agent, infectious periods, incubation period, susceptibility, and resistance [1]. Some devastating infectious diseases like Lassa fever are endemic in many parts of the world and continue to emerge.

Lassa fever, also known as Lassa hemorrhagic fever (LHF), is an animal-borne, or zoonotic, acute viral hemorrhagic illness caused by Lassa virus, a member of the family Arenaviridae. The reservoir is the rats Mastomys natalensis, M. erythroleucus, and Hylomyscus pamfi. Food contaminated with rodent urine, saliva, or feces is the main cause of human cases. Human-to-human transmission can occur via exposure to the blood, feces, urine, saliva, or vomitus of infected patients $[2,3]$. The incubation period is ca. 2-21 days. LHF is basically endemic in some countries of West Africa, and outbreaks have occurred in Benin, Ghana,
Guinea, Liberia, Nigeria, Sierra Leone, and Togo. Cases have been imported to Germany, United Kingdom, United States, and Sweden. This disease with high mortality rates (e.g., $80 \%$ among pregnant women) is likely affecting between 100,000 and 300,000 people every year, and it kills around 5,000 people, almost all in West Africa alone $[4,5]$.

Fractional Calculus (FC) is a fruitful field of mathematical research with numerous applications in engineering [6], nanotechnology [7], optics [8], human diseases [9], and chaos soliton theory [10]. Application of FC is also adopted in biology, heat transfer, system identification, genetic algorithms, traffic systems, telecommunications, physics as well as finance, and economics [11-20].

Herein, we are interested in the paradigm showing the nature of LHF, since it is lethal and transmissible $[1,2,21$, 22]. Using mathematical modeling to probe this fatal illness may provide insight, answers, and guidance useful for controlling its spread, and the originating applications. The given model is studied through Caputo fractional operator. In this model, the Caputo fractional operator is used since 
it permits both initial and boundary conditions to involve in modeling problems. The Caputo fractional operator can be applied on sufficiently differentiable functions only. One of the advantages of the fractional derivative of Caputo compared with the fractional derivative 3 of Riemann-Liouville is that the fractional derivative of Caputo for the constant is equal to zero. We develop an approximate solution to the fractional-order model describing the LHF affecting pregnant women using the Laplace transform coupled with the ADM. Numerical simulations are also presented. A fractional optimal control for the SIRD model is ultimately offered. For a good survey on the approximating of the differential equations, see [23-26]. Some recent research fractional optimal controls can be found in [27-29]. Atangana [21] studied the LHF using beta differential operator, where the model was proposed as follows in (1). Table 1 shows the meaning of the parameters in the model (1).

$$
\begin{aligned}
& { }_{0}^{A} D_{t}^{\beta} S(t)=-u_{1} S(t) I(t)+(\mu-l) N+\omega R(t)+\left(u_{2}-l\right) S(t), \\
& { }_{0}^{A} D_{t}^{\beta} I(t)=u_{1} S(t) I(t)-\left(\omega+u_{2}\right) I(t)-u_{2} S(t), \\
& { }_{0}^{A} D_{t}^{\beta} R(t)=u_{2} I(t)-\omega R(t), \\
& { }_{0}^{A} D_{t}^{\beta} D(t)=\omega I(t)+(l-\mu) N+l S(t),
\end{aligned}
$$

where ${ }_{0}^{A} D_{t}^{\beta} g(t)=(t+1 / \Gamma(\beta))^{1-\beta} \lim _{h \rightarrow 0} g(t+h)-g(t) / h$.

Also, the model has been studied by Goyal et al. [22]. The $q$-homotopy analysis transform method ( $q$-HATM) has been applied for solving the LHF model [30]. The LHF model via Atangana-Baleanu fractional derivative has been studied in [31]. The SIR model has been studied through Caputo-Fabrizio fractional operator in [32].

In this paper, we reconsider the system (1) by the following fractional sense of the system (1)

$$
\begin{aligned}
& D^{\left(\alpha_{1}\right)} S(t)=-u_{1} S I+(\mu-l) N+\omega R+\left(u_{2}-l\right) S \\
& D^{\left(\alpha_{2}\right)} I(t)=u_{1} S I-\left(\omega+u_{2}\right) I-u_{2} S \\
& D^{\left(\alpha_{3}\right)} R(t)=u_{2} I-\omega R \\
& D^{\left(\alpha_{4}\right)} D(t)=\omega I+(l-\mu) N+l S
\end{aligned}
$$

where $D^{(r)}$ is the Caputo fractional derivative of order $r$.

We organize the rest of our paper as follows. In Section 2 , we provide some basic mathematical concepts that will be necessary for our work. Also, the description of the proposed model is presented. We then follow by presenting

\begin{tabular}{|c|c|}
\hline Parameters & Meaning \\
\hline$S(t)$ & Susceptible pregnant women population \\
\hline$I(t)$ & Infected pregnant women population \\
\hline$R(t)$ & Recovery of pregnant women population \\
\hline$D(t)$ & $\begin{array}{l}\text { The population of pregnant women dying } \\
\text { in that country }\end{array}$ \\
\hline$u_{1}$ & The rate at which the women are infected \\
\hline$N$ & $\begin{array}{l}\text { The total number of adult women in the } \\
\text { given country }\end{array}$ \\
\hline$\mu$ & The rate at which the women are pregnant \\
\hline$\omega$ & The rate of death of pregnant women with LHF \\
\hline$l$ & $\begin{array}{l}\text { The rate of death of pregnant women with } \\
\text { another disease or with natural death }\end{array}$ \\
\hline$u_{2}$ & The rate of recovery of pregnant women from $\mathrm{LHF}$ \\
\hline
\end{tabular}
the series solution for the proposed model in Section 3. We provide the approximate solution construction in Section 4 . The numerical solution of the proposed model, having arbitrary order, is given in Section 5. The optimal control case is studied in Section 6. In Section 7, a concise conclusion of our paper results is presented.
TABLE 1: The meaning of the parameters in the model (1).

\section{Preliminaries}

For studying the proposed model (2), we present in this section the basic definitions concerned with fractional calculus. For a good survey on the basic definitions and several applications on fractional calculus, see $[6,7,33]$.

\subsection{Some Important Definitions}

Definition 1. The Riemann-Liouville integral is represented by

$$
I^{\alpha} z(t)=\frac{1}{\Gamma(\alpha)} \int_{0}^{t}(t-x)^{\alpha-1} z(x) d x,
$$

where $\Gamma$ is the known gamma function and $\alpha \in(0,1)$ is the order of this fractional integral.

Definition 2. The Caputo-type fractional derivative of order $\alpha$ is defined as

$$
D^{(\alpha)} z(t)=\frac{1}{\Gamma(k-\alpha)} \int_{0}^{t}(t-x)^{k-\alpha-1} z^{(k)}(x) d x
$$

Definition 3. The Laplace transform of Caputo fractional derivative of order $\alpha$ [33] is given by

$$
\begin{aligned}
\mathscr{L}\left\{D^{(\alpha)} z(t)\right\} & =s^{\alpha} Z(s)-\sum_{i=0}^{m-1} s^{\alpha-i-1} z^{(i)}(0) ; m-1<\alpha \\
& <m, m \in N ; Z(s)=\mathscr{L}\{z(t)\} .
\end{aligned}
$$

2.2. Mathematical Formulation and Description for the Proposed Model. The meaning of the parameters is shown in Table 1. Now, the description and the mathematical formulation of the model can be shown as follows.

With time $t$, we can express the rate of change in susceptible population as follows

$$
D^{\left(\alpha_{1}\right)} S(t)=-u_{1} S I+(\mu-l) N+\omega R+\left(u_{2}-l\right) S .
$$




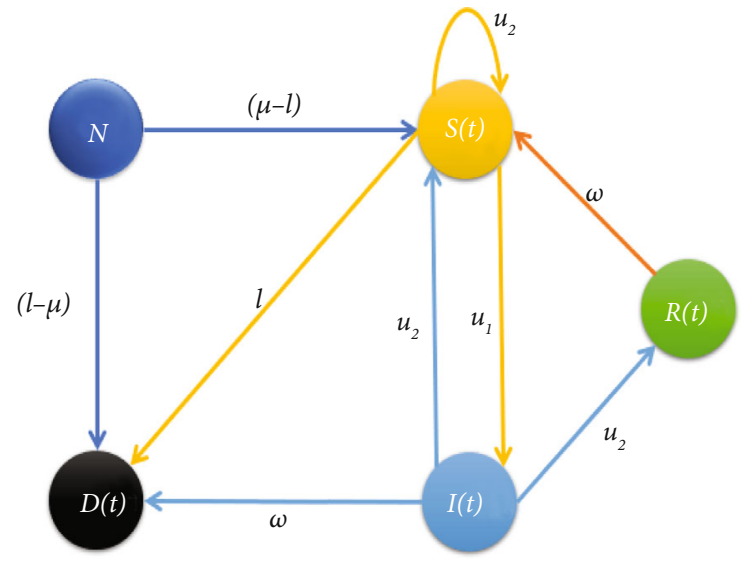

FIgURE 1: Signal flow graph of the studied LHF model.

With time $t$, we can express the rate of change in the population of the infected pregnant women as follows

$$
D^{\left(\alpha_{2}\right)} I(t)=u_{1} S I-\left(\omega+u_{2}\right) I-u_{2} S .
$$

Here, $u_{1} S I$ is the number of pregnant women excluded from the group of susceptible.

With time $t$, the rate of change in recovery population is written as

$$
D^{\left(\alpha_{3}\right)} R(t)=u_{2} I-\omega R
$$

Finally, the rate of change of dying population with time $t$ is given as

$$
D^{\left(\alpha_{4}\right)} D(t)=\omega I+(l-\mu) N+l S .
$$

Hence, the mathematical model for the LHF is given by model (2), with initial conditions

$$
S(0)=S_{0}, I(0)=I_{0}, R(0)=R_{0}, D(0)=D_{0} .
$$

Also $0<\alpha_{i}<1, i \in\{1,2,3,4\}$. Here, $S(t), I(t), R(t)$, and $D(t)$ are sufficiently differentiable functions. Model (2) has not yet been studied by using the Laplace transform coupled with the ADM. Also, we introduce numerical simulations and fractional optimal controls for the SIRD model.

The interaction between different compartments of LHF model (2) can be viewed via the following signal flow graph shown in Figure 1.

2.3. Calculating the Basic Reproduction Number $R_{0}$. Basic reproduction number $R_{0}$ is the expected production of newly infected females, in a totally susceptible women, by meeting a typical infective woman. The local stability and instability of the disease-free equilibrium (DFE) depend on the value of $R_{0}$. Also, it defines the local stability threshold for the DFE. Moreover, it is a very important tool for controlling the disease, and it is an essential epidemiological criteria of disease. When $R_{0}<1$, the DFE is locally asymptotically sta- ble; a small number of infections into the population may cause it to evolve into an endemic prevalence. From another point of view, when $R_{0}>1$, the DFE is locally unstable; a sufficiently small number of infected women will produce an outbreak. Here, $R_{0}$ is estimated from the paradigm of nonlinear FDE's (2) via the next-generation matrix method [34].

Since the Jacobian matrix is

$$
J=\left(\begin{array}{cccc}
-u_{1} I+\left(u_{2}-l\right) & -u_{1} S & \omega & 0 \\
u_{1} I-u_{2} & u_{1} S-\omega-u_{2} & 0 & 0 \\
0 & u_{2} & -\omega & 0 \\
l & \omega & 0 & 0
\end{array}\right) .
$$

At the disease-free equilibrium point $(\mu-l / l N, 0,0,0)$ and $u_{2}=0$ then by putting the model in the $F-V$ form [34] where

$$
F=\left(\begin{array}{cc}
\frac{u_{1}(\mu-l) N}{l} & 0 \\
0 & 0
\end{array}\right), V=\left(\begin{array}{cc}
\omega & 0 \\
0 & \omega
\end{array}\right), V^{-1}=\left(\begin{array}{cc}
\frac{1}{\omega} & 0 \\
0 & \frac{1}{\omega}
\end{array}\right) \text {, }
$$

then

$$
F V^{-1}=\left(\begin{array}{cc}
\frac{u_{1}(\mu-l) N}{l \omega} & 0 \\
0 & 0
\end{array}\right) .
$$

Then, the basic reproduction number of the given paradigm is $R_{0}=u_{1}(\mu-l) / \omega l N$. If $R_{0}<1$, then, infection cannot develop because an infected woman infects less than one new woman during their infectious period on average. Conversely, if $R_{0}>1$, the disease can enter the population because on average each infected woman will infect more than one new woman.

\section{Series Solution for the Proposed Model}

Applying Laplace transformation of (2) gives

$$
\left\{\begin{array}{l}
\mathscr{L}\left\{D^{\left(\alpha_{1}\right)} S(t)\right\}=\mathscr{L}\left\{-u_{1} S I+(\mu-l) N+\omega R+\left(u_{2}-l\right) S\right\} \\
\mathscr{L}\left\{D^{\left(\alpha_{2}\right)} I(t)\right\}=\mathscr{L}\left\{u_{1} S I-\left(\omega+u_{2}\right) I-u_{2} S\right\} \\
\mathscr{L}\left\{D^{\left(\alpha_{3}\right)} R(t)\right\}=\mathscr{L}\left\{u_{2} I-\omega R\right\} \\
\mathscr{L}\left\{D^{\left(\alpha_{4}\right)} D(t)\right\}=\mathscr{L}\{\omega I+(l-\mu) N+l S\}
\end{array}\right.
$$


which gives

$$
\left\{\begin{array}{l}
s^{\alpha_{1}} S^{*}(s)-s^{\alpha_{1}-1} S(0)=\mathscr{L}\left\{-u_{1} S I+(\mu-l) N+\omega R+\left(u_{2}-l\right) S\right\} \\
s^{\alpha_{2}} I^{*}(s)-s^{\alpha_{2}-1} I(0)=\mathscr{L}\left\{u_{1} S I-\left(\omega+u_{2}\right) I-u_{2} S\right\} \\
s^{\alpha_{3}} R^{*}(s)-s^{\alpha_{3}-1} R(0)=\mathscr{L}\left\{u_{2} I-\omega R\right\} \\
s^{\alpha_{4}} D^{*}(s)-s^{\alpha_{4}-1} D(0)=\mathscr{L}\{\omega I+(l-\mu) N+l S\}
\end{array}\right.
$$

where $S^{*}(s)=\mathscr{L}\{S(t)\}, I^{*}(s)=\mathscr{L}\{I(t)\}, R^{*}(s)=\mathscr{L}\{R(t)\}$, $D^{*}(s)=\mathscr{L}\{D(t)\}$.

By dividing the $i$ th equation of (18) by $s^{\alpha_{i}}$, we get

$$
\left\{\begin{array}{l}
S^{*}(s)=\frac{1}{s} S(0)+\frac{1}{s^{\alpha_{1}}} \mathscr{L}\left\{-u_{1} S I+(\mu-l) N+\omega R+\left(u_{2}-l\right) S\right\}, \\
I^{*}(s)=\frac{1}{s} I(0)+\frac{1}{s^{\alpha_{2}}} \mathscr{L}\left\{u_{1} S I-\left(\omega+u_{2}\right) I-u_{2} S\right\}, \\
R^{*}(s)=\frac{1}{s} R(0)+\frac{1}{s^{\alpha_{3}}} \mathscr{L}\left\{u_{2} I-\omega R\right\}, \\
D^{*}(s)=\frac{1}{s} D(0)+\frac{1}{s^{\alpha_{4}}} \mathscr{L}\{\omega I+(l-\mu) N+l S\} .
\end{array}\right.
$$

Taking the inverse of Laplace transformation on (19) gives

$$
\left\{\begin{array}{l}
S(t)=S(0)+\mathscr{L}^{-1}\left[\frac{1}{s^{\alpha_{1}}} \mathscr{L}\left\{-u_{1} S I+(\mu-l) N+\omega R+\left(u_{2}-l\right) S\right\}\right] \\
I(t)=I(0)+\mathscr{L}^{-1}\left[\frac{1}{s^{\alpha_{2}}} \mathscr{L}\left\{u_{1} S I-\left(\omega+u_{2}\right) I-u_{2} S\right\}\right] \\
R(t)=R(0)+\mathscr{L}^{-1}\left[\frac{1}{s^{\alpha_{3}}} \mathscr{L}\left\{u_{2} I-\omega R\right\}\right] \\
D(t)=D(0)+\mathscr{L}^{-1}\left[\frac{1}{s^{\alpha_{4}}} \mathscr{L}\{\omega I+(l-\mu) N+l S\}\right] .
\end{array}\right.
$$

Now, we will find the series solution by putting

$$
\begin{aligned}
& S(t)=\sum_{k=0}^{\infty} S_{k}(t), I(t)=\sum_{k=0}^{\infty} I_{k}(t), \\
& R(t)=\sum_{k=0}^{\infty} R_{k}(t), D(t)=\sum_{k=0}^{\infty} D_{k}(t) .
\end{aligned}
$$

Decomposing the terms $S(t) I(t)$ gives

$$
S(t) I(t)=\sum_{k=0}^{\infty} G_{k}(t)
$$

The polynomial $G_{k}(t)$ is known as "Adomian polynomial" with the form

$$
G_{k}(t)=\frac{1}{k !}\left[\left(\frac{d}{d \varepsilon}\right)^{k}\left[\sum_{r=0}^{k} \varepsilon^{r} S_{r}(t) \sum_{r=0}^{k} \varepsilon^{r} I_{r}(t)\right]\right]_{\varepsilon=0}
$$

By substituting from (21) and (22) into (20), we get

$\mathscr{L}\left(S_{0}\right)=\frac{S_{0}}{s}, \mathscr{L}\left(I_{0}\right)=\frac{I_{0}}{s}, \mathscr{L}\left(R_{0}\right)=\frac{R_{0}}{s}, \mathscr{L}\left(D_{0}\right)=\frac{D_{0}}{s}$,

$$
\begin{aligned}
& \mathscr{L}\left(S_{1}\right)=\frac{1}{s^{\alpha_{1}}} \mathscr{L}\left\{-u_{1} G_{0}+(\mu-l) N+\omega R_{0}+\left(u_{2}-l\right) S_{0}\right\}, \\
& \mathscr{L}\left(I_{1}\right)=\frac{1}{s^{\alpha_{2}}} \mathscr{L}\left\{u_{1} G_{0}-\left(\omega+u_{2}\right) I_{0}-u_{2} S_{0}\right\}, \\
& \mathscr{L}\left(R_{1}\right)=\frac{1}{s^{\alpha_{3}}} \mathscr{L}\left\{u_{2} I_{0}-\omega R_{0}\right\}, \\
& \mathscr{L}\left(D_{1}\right)=\frac{1}{s^{\alpha_{4}}} \mathscr{L}\left\{\omega I_{0}+(l-\mu) N+l S_{0}\right\}, \\
& \mathscr{L}\left(S_{2}\right)=\frac{1}{s^{\alpha_{1}}} \mathscr{L}\left\{-u_{1} G_{1}+\omega R_{1}+\left(u_{2}-l\right) S_{1}\right\}, \\
& \mathscr{L}\left(I_{2}\right)=\frac{1}{s^{\alpha_{2}}} \mathscr{L}\left\{u_{1} G_{1}-\left(\omega+u_{2}\right) I_{1}-u_{2} S_{1}\right\}, \\
& \mathscr{L}\left(R_{2}\right)=\frac{1}{s^{\alpha_{3}}} \mathscr{L}\left\{u_{2} I_{1}-\omega R_{1}\right\}, \mathscr{L}\left(D_{2}\right)=\frac{1}{s^{\alpha_{4}}} \mathscr{L}\left\{\omega I_{1}+l S_{1}\right\},
\end{aligned}
$$

$$
\begin{aligned}
& \mathscr{L}\left(S_{3}\right)=\frac{1}{s^{\alpha_{1}}} \mathscr{L}\left\{-u_{1} G_{2}+\omega R_{2}+\left(u_{2}-l\right) S_{2}\right\}, \\
& \mathscr{L}\left(I_{3}\right)=\frac{1}{s^{\alpha_{2}}} \mathscr{L}\left\{u_{1} G_{2}-\left(\omega+u_{2}\right) I_{2}-u_{2} S_{2}\right\}, \\
& \mathscr{L}\left(R_{3}\right)=\frac{1}{s^{\alpha_{3}}} \mathscr{L}\left\{u_{2} I_{2}-\omega R_{2}\right\}, \mathscr{L}\left(D_{3}\right)=\frac{1}{s^{\alpha_{4}}} \mathscr{L}\left\{\omega I_{2}+l S_{2}\right\},
\end{aligned}
$$

$$
\begin{aligned}
& \mathscr{L}\left(S_{4}\right)=\frac{1}{s^{\alpha_{1}}} \mathscr{L}\left\{-u_{1} G_{3}+\omega R_{3}+\left(u_{2}-l\right) S_{3}\right\}, \\
& \mathscr{L}\left(I_{4}\right)=\frac{1}{s^{\alpha_{2}}} \mathscr{L}\left\{u_{1} G_{3}-\left(\omega+u_{2}\right) I_{3}-u_{2} S_{3}\right\},
\end{aligned}
$$$$
\mathscr{L}\left(R_{4}\right)=\frac{1}{s^{\alpha_{3}}} \mathscr{L}\left\{u_{2} I_{3}-\omega R_{3}\right\}, \mathscr{L}\left(D_{4}\right)=\frac{1}{s^{\alpha_{4}}} \mathscr{L}\left\{\omega I_{3}+l S_{3}\right\},
$$

$$
\mathscr{L}\left(S_{k}\right)=\frac{1}{s^{\alpha_{1}}} \mathscr{L}\left\{-u_{1} G_{k-1}+\omega R_{k-1}+\left(u_{2}-l\right) S_{k-1}\right\},
$$$$
\mathscr{L}\left(I_{k}\right)=\frac{1}{s^{\alpha_{2}}} \mathscr{L}\left\{u_{1} G_{k-1}-\left(\omega+u_{2}\right) I_{k-1}-u_{2} S_{k-1}\right\},
$$$$
\mathscr{L}\left(R_{k}\right)=\frac{1}{s^{\alpha_{3}}} \mathscr{L}\left\{u_{2} I_{k-1}-\omega R_{k-1}\right\},
$$$$
\mathscr{L}\left(D_{k}\right)=\frac{1}{s^{\alpha_{4}}} \mathscr{L}\left\{\omega I_{k-1}+l S_{k-1}\right\} \text {. }
$$

Note that $k \in\{2,3,4, . . \cdots\}$ in (29). 


\section{Approximate Solution}

4.1. Construction of the Approximate Solution. Hereafter, we will discuss an approximate solution for the proposed model. Taking the inverse of Laplace transformation for (24), (25), (26), and (27) gives

$$
\begin{aligned}
& \mathscr{L}^{-1}\left\{\frac{S_{0}}{s}\right\}=S_{0}, \mathscr{L}^{-1}\left\{\frac{I_{0}}{s}\right\}=I_{0}, \\
& \mathscr{L}^{-1}\left\{\frac{R_{0}}{s}\right\}=R_{0}, \mathscr{L}^{-1}\left\{\frac{D_{0}}{s}\right\}=D_{0} \text {, } \\
& S_{1}=a t^{\alpha_{1}}, a=\frac{-u_{1} G_{0}+(\mu-l) N+\omega R_{0}+\left(u_{2}-l\right) S_{0}}{\Gamma\left(\alpha_{1}+1\right)}, \\
& I_{1}=b t^{\alpha_{2}}, b=\frac{u_{1} G_{0}-\left(\omega+u_{2}\right) I_{0}-u_{2} S_{0}}{\Gamma\left(\alpha_{2}+1\right)}, \\
& R_{1}=c t^{\alpha_{3}}, c=\frac{u_{2} I_{0}-\omega R_{0}}{\Gamma\left(\alpha_{3}+1\right)} \\
& D_{1}=d^{1} t^{\alpha_{4}}, d^{1}=\frac{\omega I_{0}+(l-\mu) N+l S_{0}}{\Gamma\left(\alpha_{4}+1\right)}, \\
& S_{2}=d t^{2 \alpha_{1}}+e t^{\alpha_{1}+\alpha_{2}}+\mathrm{ft}^{\alpha_{1}+\alpha_{3}}, \\
& d=a\left(\left(u_{2}-l\right)-u_{1} I_{0}\right) \frac{\Gamma\left(\alpha_{1}+1\right)}{\Gamma\left(2 \alpha_{1}+1\right)}, \\
& e=-u_{1} S_{0} b \frac{\Gamma\left(\alpha_{2}+1\right)}{\Gamma\left(\alpha_{1}+\alpha_{2}+1\right)}, f=c \omega \frac{\Gamma\left(\alpha_{3}+1\right)}{\Gamma\left(\alpha_{1}+\alpha_{3}+1\right)}, \\
& I_{2}=g t^{\alpha_{1}+\alpha_{2}}+h t^{2 \alpha_{2}}, g=a\left(u_{1} I_{0}-u_{2}\right) \frac{\Gamma\left(\alpha_{1}+1\right)}{\Gamma\left(\alpha_{1}+\alpha_{2}+1\right)}, \\
& h=b\left(u_{1} S_{0}-\left(\omega+u_{2}\right)\right) \frac{\Gamma\left(\alpha_{2}+1\right)}{\Gamma\left(2 \alpha_{2}+1\right)}, \\
& R_{2}=i t^{\alpha_{2}+\alpha_{3}}+j t^{2 \alpha_{3}}, i=u_{2} b \frac{\Gamma\left(\alpha_{2}+1\right)}{\Gamma\left(\alpha_{2}+\alpha_{3}+1\right)}, \\
& j=-\omega c \frac{\Gamma\left(\alpha_{3}+1\right)}{\Gamma\left(2 \alpha_{3}+1\right)}, \\
& D_{2}=p t^{\alpha_{2}+\alpha_{4}}+q t^{\alpha_{1}+\alpha_{4}}, p=\omega b \frac{\Gamma\left(\alpha_{2}+1\right)}{\Gamma\left(\alpha_{2}+\alpha_{4}+1\right)}, \\
& q=\operatorname{la} \frac{\Gamma\left(\alpha_{1}+1\right)}{\Gamma\left(\alpha_{1}+\alpha_{4}+1\right)}, \\
& S_{3}=r t^{2 \alpha_{1}+\alpha_{2}}+s t^{3 \alpha_{1}}+r_{1} t^{\alpha_{1}+2 \alpha_{2}}+u t^{2 \alpha_{1}+\alpha_{3}} \\
& +v t^{\alpha_{1}+\alpha_{2}+\alpha_{3}}+w t^{\alpha_{1}+2 \alpha_{3}}
\end{aligned}
$$

$$
\begin{aligned}
& r=\left(-u_{1} g S_{0}-u_{1} I_{0} e-u_{1} a b+e\left(u_{2}-l\right)\right) \frac{\Gamma\left(\alpha_{1}+\alpha_{2}+1\right)}{\Gamma\left(2 \alpha_{1}+\alpha_{2}+1\right)}, \\
& s=\left(\left(-u_{1} I_{0} d\right)+d\left(u_{2}-l\right)\right) \frac{\Gamma\left(2 \alpha_{1}+1\right)}{\Gamma\left(3 \alpha_{1}+1\right)}, \\
& r_{1}=-u_{1} h S_{0} \frac{\Gamma\left(2 \alpha_{2}+1\right)}{\Gamma\left(\alpha_{1}+2 \alpha_{2}+1\right)}, \\
& \cdot u=\left(-u_{1} I_{0} f+f\left(u_{2}-l\right)\right) \frac{\Gamma\left(\alpha_{1}+\alpha_{3}+1\right)}{\Gamma\left(2 \alpha_{1}+\alpha_{3}+1\right)}, \\
& v=i \omega \frac{\Gamma\left(\alpha_{2}+\alpha_{3}+1\right)}{\Gamma\left(\alpha_{1}+\alpha_{2}+\alpha_{3}+1\right)}, w=j \omega \frac{\Gamma\left(2 \alpha_{3}+1\right)}{\Gamma\left(\alpha_{1}+2 \alpha_{3}+1\right)}, \\
& I_{3}=x t^{\alpha_{1}+2 \alpha_{2}}+y t^{3 \alpha_{2}}+z t^{2 \alpha_{1}+\alpha_{2}}+a_{1} t^{\alpha_{1}+\alpha_{2}+\alpha_{3}}, \\
& x=\left(u_{1} S_{0} g+e u_{1} I_{0}-g\left(\omega+u_{2}\right)\right. \\
& \left.+u_{1} a b-u_{2} e\right) \frac{\Gamma\left(\alpha_{1}+\alpha_{2}+1\right)}{\Gamma\left(\alpha_{1}+2 \alpha_{2}+1\right)}, \\
& y=\left(u_{1} S_{0} h-h\left(\omega+u_{2}\right)\right) \frac{\Gamma\left(2 \alpha_{2}+1\right)}{\Gamma\left(3 \alpha_{2}+1\right)} \text {, } \\
& z=\left(d u_{1} I_{0}-u_{2} d\right) \frac{\Gamma\left(2 \alpha_{1}+1\right)}{\Gamma\left(2 \alpha_{1}+\alpha_{2}+1\right)}, \\
& a_{1}=f\left(u_{1} I_{0}-u_{2}\right) \frac{\Gamma\left(\alpha_{1}+\alpha_{3}+1\right)}{\Gamma\left(\alpha_{1}+\alpha_{2}+\alpha_{3}+1\right)}, \\
& R_{3}=b_{1} t^{\alpha_{1}+\alpha_{2}+\alpha_{3}}+c_{1} t^{2 \alpha_{2}+\alpha_{3}}+d_{1} t^{\alpha_{2}+2 \alpha_{3}}+e_{1} t^{3 \alpha_{3}}, \\
& b_{1}=g u_{2} \frac{\Gamma\left(\alpha_{1}+\alpha_{2}+1\right)}{\Gamma\left(\alpha_{1}+\alpha_{2}+\alpha_{3}+1\right)}, c_{1}=h u_{2} \frac{\Gamma\left(2 \alpha_{2}+1\right)}{\Gamma\left(2 \alpha_{2}+\alpha_{3}+1\right)}, \\
& d_{1}=-\omega i \frac{\Gamma\left(\alpha_{2}+\alpha_{3}+1\right)}{\Gamma\left(\alpha_{2}+2 \alpha_{3}+1\right)}, e_{1}=-\omega j \frac{\Gamma\left(2 \alpha_{3}+1\right)}{\Gamma\left(3 \alpha_{3}+1\right)}, \\
& D_{3}=f_{1} t^{\alpha_{1}+\alpha_{2}+\alpha_{4}}+g_{1} t^{2 \alpha_{2}+\alpha_{4}}+h_{1} t^{2 \alpha_{1}+\alpha_{4}}+i_{1} t^{\alpha_{1}+\alpha_{3}+\alpha_{4}} \\
& f_{1}=(g \omega+l e) \frac{\Gamma\left(\alpha_{1}+\alpha_{2}+1\right)}{\Gamma\left(\alpha_{1}+\alpha_{2}+\alpha_{4}+1\right)}, g_{1}=\omega h \frac{\Gamma\left(2 \alpha_{2}+1\right)}{\Gamma\left(2 \alpha_{2}+\alpha_{4}+1\right)}, \\
& h_{1}=l d \frac{\Gamma\left(2 \alpha_{1}+1\right)}{\Gamma\left(2 \alpha_{1}+\alpha_{4}+1\right)}, i_{1}=l f \frac{\Gamma\left(\alpha_{1}+\alpha_{3}+1\right)}{\Gamma\left(\alpha_{1}+\alpha_{3}+\alpha_{4}+1\right)} \text {. }
\end{aligned}
$$

Since $S(t)=\sum_{k=0}^{\infty} S_{k}(t), I(t)=\sum_{k=0}^{\infty} I_{k}(t), R(t)=\sum_{k=0}^{\infty} R_{k}(t)$, $D(t)=\sum_{k=0}^{\infty} D_{k}(t)$, then, if we take the first four terms from the above series and take into consideration the equations from (30) to (46), we get

$$
\begin{aligned}
S(t)= & S_{0}+S_{1}+S_{2}+S_{3}=S_{0}+a t^{\alpha_{1}}+d t^{2 \alpha_{1}} \\
& +e t^{\alpha_{1}+\alpha_{2}}+\mathrm{ft}^{\alpha_{1}+\alpha_{3}}+r t^{2 \alpha_{1}+\alpha_{2}}+s t^{3 \alpha_{1}} \\
& +r_{1} t^{\alpha_{1}+2 \alpha_{2}}+u t^{2 \alpha_{1}+\alpha_{3}}+v t^{\alpha_{1}+\alpha_{2}+\alpha_{3}}+w t^{\alpha_{1}+2 \alpha_{3}}, \\
I(t)= & I_{0}+I_{1}+I_{2}+I_{3}=I_{0}+b t^{\alpha_{2}}+g t^{\alpha_{1}+\alpha_{2}} \\
& +h t^{2 \alpha_{2}}+x t^{\alpha_{1}+2 \alpha_{2}}+y t^{3 \alpha_{2}}+z t^{2 \alpha_{1}+\alpha_{2}}+a_{1} t^{\alpha_{1}+\alpha_{2}+\alpha_{3}},
\end{aligned}
$$




$$
\begin{aligned}
R(t)= & R_{0}+R_{1}+R_{2}+R_{3}=R_{0}+c t^{\alpha_{3}}+i t^{\alpha_{2}+\alpha_{3}}+j t^{2 \alpha_{3}} \\
& +b_{1} t^{\alpha_{1}+\alpha_{2}+\alpha_{3}}+c_{1} t^{2 \alpha_{2}+\alpha_{3}}+d_{1} t^{\alpha_{2}+2 \alpha_{3}}+e_{1} t^{3 \alpha_{3}} \\
D(t)= & D_{0}+D_{1}+D_{2}+D_{3}=D_{0}+d^{1} t^{\alpha_{4}}+p t^{\alpha_{2}+\alpha_{4}}+q t^{\alpha_{1}+\alpha_{4}} \\
& +f_{1} t^{\alpha_{1}+\alpha_{2}+\alpha_{4}}+g_{1} t^{2 \alpha_{2}+\alpha_{4}}+h_{1} t^{2 \alpha_{1}+\alpha_{4}}+i_{1} t^{\alpha_{1}+\alpha_{3}+\alpha_{4}}
\end{aligned}
$$

After the compensation by the values of $a, b, c, d^{1}, d, e, f$, $g, h, i, j, p, q, r, r_{1}, s, u, v, w, x, y, z, a_{1}, b_{1}, c_{1}, d_{1}, e_{1}, f_{1}, g_{1}, h_{1}$, $i_{1}$, we obtain $S(t)=S_{0}+S_{1}+S_{2}+S_{3}=$

$$
\begin{aligned}
& S_{0}+\left(\frac{-u_{1} G_{0}+(\mu-l) N+\omega R_{0}+\left(u_{2}-l\right) S_{0}}{\Gamma\left(\alpha_{1}+1\right)}\right) t^{\alpha_{1}}+\left(\left(\frac{-u_{1} G_{0}+(\mu-l) N+\omega R_{0}+\left(u_{2}-l\right) S_{0}}{\Gamma\left(\alpha_{1}+1\right)}\right)\left(\left(u_{2}-l\right)-u_{1} I_{0}\right) \frac{\Gamma\left(\alpha_{1}+1\right)}{\Gamma\left(2 \alpha_{1}+1\right)}\right) t^{2 \alpha_{1}} \\
& -\left(u_{1} S_{0}\left(\frac{u_{1} G_{0}-\left(\omega+u_{2}\right) I_{0}-u_{2} S_{0}}{\Gamma\left(\alpha_{2}+1\right)}\right) \frac{\Gamma\left(\alpha_{2}+1\right)}{\Gamma\left(\alpha_{1}+\alpha_{2}+1\right)}\right) t^{\alpha_{1}+\alpha_{2}}+\left(\left(\frac{u_{2} I_{0}-\omega R_{0}}{\Gamma\left(\alpha_{3}+1\right)}\right) \omega \frac{\Gamma\left(\alpha_{3}+1\right)}{\Gamma\left(\alpha_{1}+\alpha_{3}+1\right)}\right) t^{\alpha_{1}+\alpha_{3}} \\
& +\left(\begin{array}{c}
-u_{1}\left(\left(\frac{-u_{1} G_{0}+(\mu-l) N+\omega R_{0}+\left(u_{2}-l\right) S_{0}}{\Gamma\left(\alpha_{1}+1\right)}\right)\left(u_{1} I_{0}-u_{2}\right) \frac{\Gamma\left(\alpha_{1}+1\right)}{\Gamma\left(\alpha_{1}+\alpha_{2}+1\right)}\right) S_{0} \\
-u_{1} I_{0}\left(-u_{1} S_{0}\left(\frac{u_{1} G_{0}-\left(\omega+u_{2}\right) I_{0}-u_{2} S_{0}}{\Gamma\left(\alpha_{2}+1\right)}\right) \frac{\Gamma\left(\alpha_{2}+1\right)}{\Gamma\left(\alpha_{1}+\alpha_{2}+1\right)}\right) \\
-u_{1}\left(\frac{-u_{1} G_{0}+(\mu-l) N+\omega R_{0}+\left(u_{2}-l\right) S_{0}}{\Gamma\left(\alpha_{1}+1\right)}\right)\left(\frac{u_{1} G_{0}-\left(\omega+u_{2}\right) I_{0}-u_{2} S_{0}}{\Gamma\left(\alpha_{2}+1\right)}\right) \\
+\left(-u_{1} S_{0}\left(\frac{u_{1} G_{0}-\left(\omega+u_{2}\right) I_{0}-u_{2} S_{0}}{\Gamma\left(\alpha_{2}+1\right)}\right) \frac{\Gamma\left(\alpha_{2}+1\right)}{\Gamma\left(\alpha_{1}+\alpha_{2}+1\right)}\right)\left(u_{2}-l\right)
\end{array}\right) \frac{\Gamma\left(\alpha_{1}+\alpha_{2}+1\right)}{\Gamma\left(2 \alpha_{1}+\alpha_{2}+1\right)} t^{2 \alpha_{1}+\alpha_{2}} \\
& +\left(\begin{array}{c}
\left(-u_{1} I_{0}\left(\frac{-u_{1} G_{0}+(\mu-l) N+\omega R_{0}+\left(u_{2}-l\right) S_{0}}{\Gamma\left(\alpha_{1}+1\right)}\right)\left(\left(u_{2}-l\right)-u_{1} I_{0}\right) \frac{\Gamma\left(\alpha_{1}+1\right)}{\Gamma\left(2 \alpha_{1}+1\right)}\right) \\
+\left(\left(\frac{-u_{1} G_{0}+(\mu-l) N+\omega R_{0}+\left(u_{2}-l\right) S_{0}}{\Gamma\left(\alpha_{1}+1\right)}\right)\left(\left(u_{2}-l\right)-u_{1} I_{0}\right) \frac{\Gamma\left(\alpha_{1}+1\right)}{\Gamma\left(2 \alpha_{1}+1\right)}\right)\left(u_{2}-l\right)
\end{array}\right) \frac{\Gamma\left(2 \alpha_{1}+1\right)}{\Gamma\left(3 \alpha_{1}+1\right)} t^{3 \alpha_{1}}+\left(\begin{array}{c}
-u_{1} I_{0}\left(\left(\frac{u_{2} I_{0}-\omega R_{0}}{\Gamma\left(\alpha_{3}+1\right)}\right) \omega \frac{\Gamma\left(\alpha_{3}+1\right)}{\Gamma\left(\alpha_{1}+\alpha_{3}+1\right)}\right) \\
+\left(\left(\frac{u_{2} I_{0}-\omega R_{0}}{\Gamma\left(\alpha_{3}+1\right)}\right) \omega \frac{\Gamma\left(\alpha_{3}+1\right)}{\Gamma\left(\alpha_{1}+\alpha_{3}+1\right)}\right)\left(u_{2}-l\right)
\end{array}\right) \\
& \cdot \frac{\Gamma\left(\alpha_{1}+\alpha_{3}+1\right)}{\Gamma\left(2 \alpha_{1}+\alpha_{3}+1\right)} t^{2 \alpha_{1}+\alpha_{3}}+\left(u_{2}\left(\frac{u_{1} G_{0}-\left(\omega+u_{2}\right) I_{0}-u_{2} S_{0}}{\Gamma\left(\alpha_{2}+1\right)}\right) \frac{\Gamma\left(\alpha_{2}+1\right)}{\Gamma\left(\alpha_{2}+\alpha_{3}+1\right)}\right) \omega \frac{\Gamma\left(\alpha_{2}+\alpha_{3}+1\right)}{\Gamma\left(\alpha_{1}+\alpha_{2}+\alpha_{3}+1\right)} t^{\alpha_{1}+\alpha_{2}+\alpha_{3}}+\left(-\omega\left(\frac{u_{2} I_{0}-\omega R_{0}}{\Gamma\left(\alpha_{3}+1\right)}\right) \frac{\Gamma\left(\alpha_{3}+1\right)}{\Gamma\left(2 \alpha_{3}+1\right)}\right) \\
& \cdot \omega \frac{\Gamma\left(2 \alpha_{3}+1\right)}{\Gamma\left(\alpha_{1}+2 \alpha_{3}+1\right)} t^{\alpha_{1}+2 \alpha_{3}}-u_{1}\left(\left(\left(\frac{u_{1} G_{0}-\left(\omega+u_{2}\right) I_{0}-u_{2} S_{0}}{\Gamma\left(\alpha_{2}+1\right)}\right)\left(u_{1} S_{0}-\left(\omega+u_{2}\right)\right) \frac{\Gamma\left(\alpha_{2}+1\right)}{\Gamma\left(2 \alpha_{2}+1\right)}\right)\right) S_{0} \frac{\Gamma\left(2 \alpha_{2}+1\right)}{\Gamma\left(\alpha_{1}+2 \alpha_{2}+1\right)} t^{\alpha_{1}+2 \alpha_{2}} \text {, }
\end{aligned}
$$

$$
\begin{aligned}
& I(t)=I_{0}+I_{1}+I_{2}+I_{3}=I_{0}+\left(\frac{u_{1} G_{0}-\left(\omega+u_{2}\right) I_{0}-u_{2} S_{0}}{\Gamma\left(\alpha_{2}+1\right)}\right) t^{\alpha_{2}}+\left(\frac{-u_{1} G_{0}+(\mu-l) N+\omega R_{0}+\left(u_{2}-l\right) S_{0}}{\Gamma\left(\alpha_{1}+1\right)}\right) \\
& \cdot\left(u_{1} I_{0}-u_{2}\right) \frac{\Gamma\left(\alpha_{1}+1\right)}{\Gamma\left(\alpha_{1}+\alpha_{2}+1\right)} t^{\alpha_{1}+\alpha_{2}}+\left(\left(\frac{u_{1} G_{0}-\left(\omega+u_{2}\right) I_{0}-u_{2} S_{0}}{\Gamma\left(\alpha_{2}+1\right)}\right)\left(u_{1} S_{0}-\left(\omega+u_{2}\right)\right) \frac{\Gamma\left(\alpha_{2}+1\right)}{\Gamma\left(2 \alpha_{2}+1\right)}\right) t^{2 \alpha_{2}} \\
& \left.+\left(\begin{array}{c}
u_{1} S_{0}\left(\left(\frac{-u_{1} G_{0}+(\mu-l) N+\omega R_{0}+\left(u_{2}-l\right) S_{0}}{\Gamma\left(\alpha_{1}+1\right)}\right)\left(u_{1} I_{0}-u_{2}\right) \frac{\Gamma\left(\alpha_{1}+1\right)}{\Gamma\left(\alpha_{1}+\alpha_{2}+1\right)}\right) \\
+\left(-u_{1} S_{0}\left(\frac{u_{1} G_{0}-\left(\omega+u_{2}\right) I_{0}-u_{2} S_{0}}{\Gamma\left(\alpha_{2}+1\right)}\right) \frac{\Gamma\left(\alpha_{2}+1\right)}{\Gamma\left(\alpha_{1}+\alpha_{2}+1\right)}\right) u_{1} I_{0} \\
-\left(\left(\frac{-u_{1} G_{0}+(\mu-l) N+\omega R_{0}+\left(u_{2}-l\right) S_{0}}{\Gamma\left(\alpha_{1}+1\right)}\right)\left(u_{1} I_{0}-u_{2}\right) \frac{\Gamma\left(\alpha_{1}+1\right)}{\Gamma\left(\alpha_{1}+\alpha_{2}+1\right)}\right)\left(\omega+u_{2}\right) \\
+u_{1}\left(\frac{-u_{1} G_{0}+(\mu-l) N+\omega R_{0}+\left(u_{2}-l\right) S_{0}}{\Gamma\left(\alpha_{1}+1\right)}\right)\left(\frac{u_{1} G_{0}-\left(\omega+u_{2}\right) I_{0}-u_{2} S_{0}}{\Gamma\left(\alpha_{2}+1\right)}\right) \\
-u_{2}\left(-u_{1} S_{0}\left(\frac{u_{1} G_{0}-\left(\omega+u_{2}\right) I_{0}-u_{2} S_{0}}{\Gamma\left(\alpha_{2}+1\right)}\right) \frac{\Gamma\left(\alpha_{2}+1\right)}{\Gamma\left(\alpha_{1}+\alpha_{2}+1\right)}\right)
\end{array}\right) \quad \frac{\Gamma\left(\alpha_{1}+\alpha_{2}+1\right)}{\Gamma\left(\alpha_{1}+2 \alpha_{2}+1\right)}\right) t^{\alpha_{1}+2 \alpha_{2}} \\
& \left.+\left(\begin{array}{c}
u_{1} S_{0}\left(\left(\frac{u_{1} G_{0}-\left(\omega+u_{2}\right) I_{0}-u_{2} S_{0}}{\Gamma\left(\alpha_{2}+1\right)}\right)\left(u_{1} S_{0}-\left(\omega+u_{2}\right)\right) \frac{\Gamma\left(\alpha_{2}+1\right)}{\Gamma\left(2 \alpha_{2}+1\right)}\right) \\
-\left(\left(\frac{u_{1} G_{0}-\left(\omega+u_{2}\right) I_{0}-u_{2} S_{0}}{\Gamma\left(\alpha_{2}+1\right)}\right)\left(u_{1} S_{0}-\left(\omega+u_{2}\right)\right) \frac{\Gamma\left(\alpha_{2}+1\right)}{\Gamma\left(2 \alpha_{2}+1\right)}\right)\left(\omega+u_{2}\right)
\end{array}\right) \frac{\Gamma\left(2 \alpha_{2}+1\right)}{\Gamma\left(3 \alpha_{2}+1\right)}\right) t^{3 \alpha_{2}} \\
& +\left(\left(u_{1} I_{0}-u_{2}\right)\left(\left(\frac{-u_{1} G_{0}+(\mu-l) N+\omega R_{0}+\left(u_{2}-l\right) S_{0}}{\Gamma\left(\alpha_{1}+1\right)}\right)\left(\left(u_{2}-l\right)-u_{1} I_{0}\right) \frac{\Gamma\left(\alpha_{1}+1\right)}{\Gamma\left(2 \alpha_{1}+1\right)}\right) \frac{\Gamma\left(2 \alpha_{1}+1\right)}{\Gamma\left(2 \alpha_{1}+\alpha_{2}+1\right)}\right) t^{2 \alpha_{1}+\alpha_{2}} \\
& +\left(\left(\left(\frac{u_{2} I_{0}-\omega R_{0}}{\Gamma\left(\alpha_{3}+1\right)}\right) \omega \frac{\Gamma\left(\alpha_{3}+1\right)}{\Gamma\left(\alpha_{1}+\alpha_{3}+1\right)}\right)\left(u_{1} I_{0}-u_{2}\right) \frac{\Gamma\left(\alpha_{1}+\alpha_{3}+1\right)}{\Gamma\left(\alpha_{1}+\alpha_{2}+\alpha_{3}+1\right)}\right) t^{\alpha_{1}+\alpha_{2}+\alpha_{3}},
\end{aligned}
$$




$$
\begin{aligned}
& R(t)=R_{0}+R_{1}+R_{2}+R_{3}=R_{0}+\left(\frac{u_{2} I_{0}-\omega R_{0}}{\Gamma\left(\alpha_{3}+1\right)}\right) t^{\alpha_{3}}+\left(u_{2}\left(\frac{u_{1} G_{0}-\left(\omega+u_{2}\right) I_{0}-u_{2} S_{0}}{\Gamma\left(\alpha_{2}+1\right)}\right) \frac{\Gamma\left(\alpha_{2}+1\right)}{\Gamma\left(\alpha_{2}+\alpha_{3}+1\right)}\right) t^{\alpha_{2}+\alpha_{3}} \\
& -\left(\omega\left(\frac{u_{2} I_{0}-\omega R_{0}}{\Gamma\left(\alpha_{3}+1\right)}\right) \frac{\Gamma\left(\alpha_{3}+1\right)}{\Gamma\left(2 \alpha_{3}+1\right)}\right) t^{2 \alpha_{3}}+\left(\left(\frac{-u_{1} G_{0}+(\mu-l) N+\omega R_{0}+\left(u_{2}-l\right) S_{0}}{\Gamma\left(\alpha_{1}+1\right)}\right)\left(u_{1} I_{0}-u_{2}\right) \frac{\Gamma\left(\alpha_{1}+1\right)}{\Gamma\left(\alpha_{1}+\alpha_{2}+1\right)}\right) \\
& \cdot u_{2} \frac{\Gamma\left(\alpha_{1}+\alpha_{2}+1\right)}{\Gamma\left(\alpha_{1}+\alpha_{2}+\alpha_{3}+1\right)} t^{\alpha_{1}+\alpha_{2}+\alpha_{3}}+\left(\left(\frac{u_{1} G_{0}-\left(\omega+u_{2}\right) I_{0}-u_{2} S_{0}}{\Gamma\left(\alpha_{2}+1\right)}\right)\left(u_{1} S_{0}-\left(\omega+u_{2}\right)\right) \frac{\Gamma\left(\alpha_{2}+1\right)}{\Gamma\left(2 \alpha_{2}+1\right)}\right) \\
& \cdot u_{2} \frac{\Gamma\left(2 \alpha_{2}+1\right)}{\Gamma\left(2 \alpha_{2}+\alpha_{3}+1\right)} t^{2 \alpha_{2}+\alpha_{3}}-\omega\left(u_{2}\left(\frac{u_{1} G_{0}-\left(\omega+u_{2}\right) I_{0}-u_{2} S_{0}}{\Gamma\left(\alpha_{2}+1\right)}\right) \frac{\Gamma\left(\alpha_{2}+1\right)}{\Gamma\left(\alpha_{2}+\alpha_{3}+1\right)}\right) \frac{\Gamma\left(\alpha_{2}+\alpha_{3}+1\right)}{\Gamma\left(\alpha_{2}+2 \alpha_{3}+1\right)} t^{\alpha_{2}+2 \alpha_{3}} \\
& -\omega\left(-\omega\left(\frac{u_{2} I_{0}-\omega R_{0}}{\Gamma\left(\alpha_{3}+1\right)}\right) \frac{\Gamma\left(\alpha_{3}+1\right)}{\Gamma\left(2 \alpha_{3}+1\right)}\right) \frac{\Gamma\left(2 \alpha_{3}+1\right)}{\Gamma\left(3 \alpha_{3}+1\right)} t^{3 \alpha_{3}}, \\
& D(t)=D_{0}+D_{1}+D_{2}+D_{3}=D_{0}+\left(\frac{\omega I_{0}+(l-\mu) N+l S_{0}}{\Gamma\left(\alpha_{4}+1\right)}\right) t^{\alpha_{4}}+\left(\omega\left(\frac{u_{1} G_{0}-\left(\omega+u_{2}\right) I_{0}-u_{2} S_{0}}{\Gamma\left(\alpha_{2}+1\right)}\right) \frac{\Gamma\left(\alpha_{2}+1\right)}{\Gamma\left(\alpha_{2}+\alpha_{4}+1\right)}\right) t^{\alpha_{2}+\alpha_{4}} \\
& +\left(l\left(\frac{-u_{1} G_{0}+(\mu-l) N+\omega R_{0}+\left(u_{2}-l\right) S_{0}}{\Gamma\left(\alpha_{1}+1\right)}\right) \frac{\Gamma\left(\alpha_{1}+1\right)}{\Gamma\left(\alpha_{1}+\alpha_{4}+1\right)}\right) t^{\alpha_{1}+\alpha_{4}} \\
& +\left(\begin{array}{c}
\left(\left(\frac{-u_{1} G_{0}+(\mu-l) N+\omega R_{0}+\left(u_{2}-l\right) S_{0}}{\Gamma\left(\alpha_{1}+1\right)}\right)\left(u_{1} I_{0}-u_{2}\right) \frac{\Gamma\left(\alpha_{1}+1\right)}{\Gamma\left(\alpha_{1}+\alpha_{2}+1\right)}\right) \omega \\
+l\left(-u_{1} S_{0}\left(\frac{u_{1} G_{0}-\left(\omega+u_{2}\right) I_{0}-u_{2} S_{0}}{\Gamma\left(\alpha_{2}+1\right)}\right) \frac{\Gamma\left(\alpha_{2}+1\right)}{\Gamma\left(\alpha_{1}+\alpha_{2}+1\right)}\right)
\end{array}\right) \frac{\Gamma\left(\alpha_{1}+\alpha_{2}+1\right)}{\Gamma\left(\alpha_{1}+\alpha_{2}+\alpha_{4}+1\right)} t^{\alpha_{1}+\alpha_{2}+\alpha_{4}} \\
& +\omega\left(\left(\frac{u_{1} G_{0}-\left(\omega+u_{2}\right) I_{0}-u_{2} S_{0}}{\Gamma\left(\alpha_{2}+1\right)}\right)\left(u_{1} S_{0}-\left(\omega+u_{2}\right)\right) \frac{\Gamma\left(\alpha_{2}+1\right)}{\Gamma\left(2 \alpha_{2}+1\right)}\right) \frac{\Gamma\left(2 \alpha_{2}+1\right)}{\Gamma\left(2 \alpha_{2}+\alpha_{4}+1\right)} t^{2 \alpha_{2}+\alpha_{4}} \\
& +l\left(\left(\frac{-u_{1} G_{0}+(\mu-l) N+\omega R_{0}+\left(u_{2}-l\right) S_{0}}{\Gamma\left(\alpha_{1}+1\right)}\right)\left(\left(u_{2}-l\right)-u_{1} I_{0}\right) \frac{\Gamma\left(\alpha_{1}+1\right)}{\Gamma\left(2 \alpha_{1}+1\right)}\right) \frac{\Gamma\left(2 \alpha_{1}+1\right)}{\Gamma\left(2 \alpha_{1}+\alpha_{4}+1\right)} t^{2 \alpha_{1}+\alpha_{4}} \\
& +l\left(\left(\frac{u_{2} I_{0}-\omega R_{0}}{\Gamma\left(\alpha_{3}+1\right)}\right) \omega \frac{\Gamma\left(\alpha_{3}+1\right)}{\Gamma\left(\alpha_{1}+\alpha_{3}+1\right)}\right) \frac{\Gamma\left(\alpha_{1}+\alpha_{3}+1\right)}{\Gamma\left(\alpha_{1}+\alpha_{3}+\alpha_{4}+1\right)} t^{\alpha_{1}+\alpha_{3}+\alpha_{4}} .
\end{aligned}
$$

Now, by taking the first three terms of $S(t), I(t), R(t)$, and $D(t)$ and putting $\alpha_{1}=\alpha_{2}=\alpha_{3}=\alpha_{4}=\alpha, u_{1}=0.4, \mu=0.3$, $N=1000, l=0.2, \omega=0.8, \quad u_{2}=0.2, S(0)=900, I(0)=10$, $R(0)=D(0)=0$, in the proposed model yield (see [30]).

$$
\begin{aligned}
& S(t)=S_{0}+S_{1}+S_{2}=900-\frac{3500}{\Gamma(\alpha+1)} t^{\alpha}-\frac{1213598.4}{\Gamma(2 \alpha+1)} t^{2 \alpha}, \\
& I(t)=I_{0}+I_{1}+I_{2}=10+\frac{3410}{\Gamma(\alpha+1)} t^{\alpha}+\frac{1210890}{\Gamma(2 \alpha+1)} t^{2 \alpha}, \\
& R(t)=R_{0}+R_{1}+R_{2}=0+\frac{2}{\Gamma(\alpha+1)} t^{\alpha}+\frac{680.4}{\Gamma(2 \alpha+1)} t^{2 \alpha}, \\
& D(t)=D_{0}+D_{1}+D_{2}=0+\frac{88}{\Gamma(\alpha+1)} t^{\alpha}+\frac{2028}{\Gamma(2 \alpha+1)} t^{2 \alpha} .
\end{aligned}
$$

Figure 2 exhibits the dynamical behavior of $S(t), I(t)$, $R(t)$, and $D(t)$ against various fractional orders $(\alpha=0,7$; $0.8 ; 0.9 ; 1)$.

4.2. Convergence and Error Analysis. It is clear that the previous solution is a series. This solution converges rapidly and uniformly to the exact solution. We will use classical techniques to prove the convergence of the series (21), and hence, we can get the sufficient conditions for the convergence of the used method.
Theorem 4. Suppose $\wp$ is the Banach space and $\sigma: \wp \longrightarrow \wp$ is a contractive nonlinear operator with for all $x, x^{\prime} \in \wp, \| \sigma(x)$ $-\sigma\left(x^{\prime}\right)\|\leq \lambda\| x-x^{\prime} \|, 0<\lambda<1$. Then, based on Banach contraction principle, there is a unique point $x$ such that $\sigma x=x$, where $x=(s, i, r, d)$. By applying the ADM, the series in (21) can be written as follows:

$$
x_{n}=\sigma x_{n-1}, x_{n-1}=\sum_{k=1}^{n-1} x_{k}, n=1,2,3, \cdots
$$

and suppose that $x_{0}=x_{0} \in A_{j}(x)$ where $A_{j}(x)=\left\{x^{\prime} \in \wp: \| x\right.$ $\left.-x^{\prime} \|<j\right\}$, then, we obtain

$$
\begin{aligned}
& x_{n} \in A_{j}(x) ; \\
& \lim _{n \longrightarrow \infty} x_{n}=x .
\end{aligned}
$$

Proof. For the first part $(a) x_{n} \in A_{j}(x)$; by the mathematical induction if $n=1$, we obtain

$$
\left\|x_{0}-x\right\|=\left\|\sigma\left(x_{0}\right)-\sigma(x)\right\| \leq \lambda\left\|x_{0}-x\right\| .
$$

Suppose the result is true at $n-1$, hence,

$$
\left\|x_{0}-x\right\| \leq \lambda^{n-1}\left\|x_{0}-x\right\|
$$



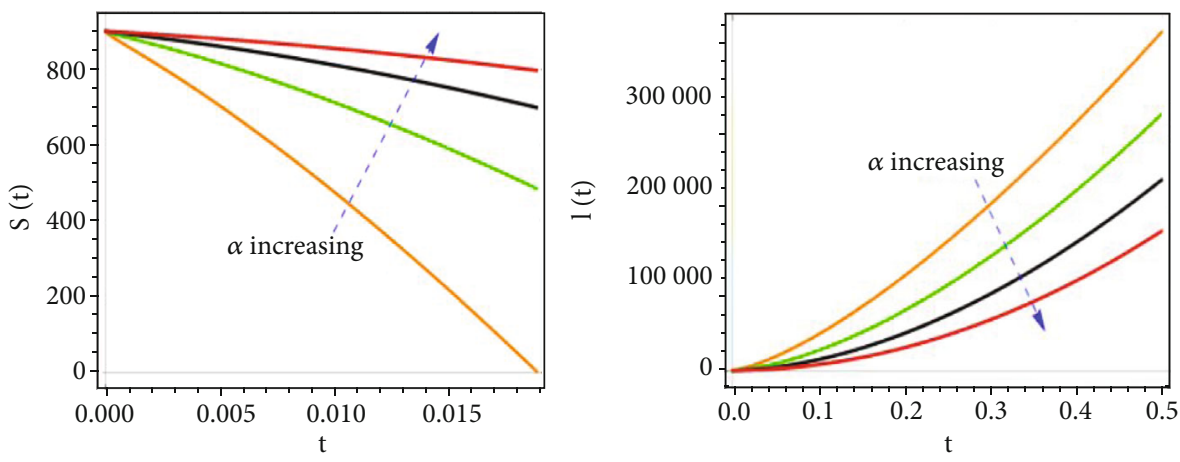

$$
\begin{aligned}
\alpha & =0.7 \\
\alpha & =0.8 \\
\alpha & =0.9 \\
\alpha & =1
\end{aligned}
$$
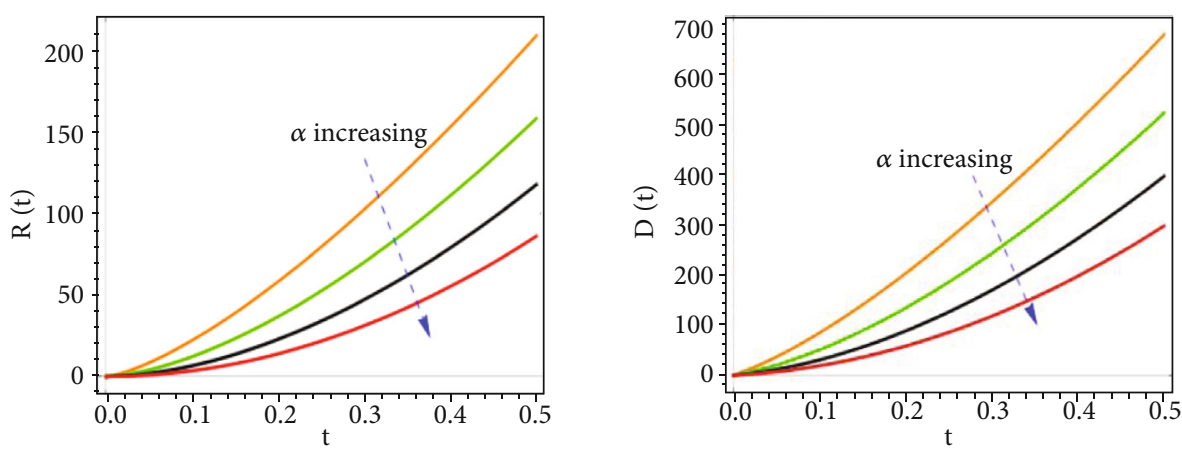

$$
\begin{aligned}
\alpha & =0.7 \\
\alpha & =0.8 \\
\square & =0.9 \\
\square & =1
\end{aligned}
$$

$$
\begin{aligned}
\alpha & =0.7 \\
\alpha & =0.8 \\
\alpha & =0.9 \\
\alpha & =1
\end{aligned}
$$

FIgure 2: The behavior of $S(t), I(t), R(t)$, and $D(t)$ populations versus time with different fractional derivative orders.

We have

$$
\begin{aligned}
\left\|x_{n}-x\right\| & =\left\|\sigma\left(x_{n-1}\right)-\sigma(x)\right\| \leq \lambda\left\|x_{n-1}-x\right\| \\
& \leq \lambda^{n}\left\|x_{0}-x\right\|, \\
\left\|x_{n}-x\right\| & \leq \lambda^{n}\left\|x_{0}-x\right\| \leq \lambda^{n} j<j .
\end{aligned}
$$

This shows that $x_{n} \in A_{j}(x)$.

For the second part (b) $\lim _{n \rightarrow \infty} x_{n}=x$.

Since $\left\|x_{n}-x\right\| \leq \lambda^{n}\left\|x_{0}-x\right\|$ and $\lim _{n \rightarrow \infty} \lambda^{n}=0$. So, we obtain $\lim _{n \longrightarrow \infty}\left\|x_{n}-x\right\|=0 \Rightarrow \lim _{n \longrightarrow \infty} x_{n}=x$.

\section{Fractional-Order Numerical Simulations of the SIRD System}

First, we recall the basics of the applied numerical technique that have been given for the numerical simulation of fractional IVPs with Caputo derivatives (2). The technique is an extension of the well-known Adams-Bashforth-Moulton (ABM) integrator which is common for the numerical simulation of $1^{\text {st }}$ order differential equations [35]. This tech- nique comes from the idea that the IVP is equal to the equation of Volterra integral. The fractional ABM technique is fully presented by the following formulas (all other states can be found same as $S$ ). Assuming that $[0, T]$ is the domain of the solution and $n=0,1,2, \cdots, N, h=T / N, t_{n}=n h$ :

$$
\begin{aligned}
S\left(t_{n+1}\right)= & \sum_{i=0}^{\lceil\alpha\rceil-1} S_{0}^{(i)} \frac{t_{n+1}^{i+1}}{i !}+\frac{h^{\alpha}}{\Gamma(\alpha+2)} \psi_{1}\left(t_{n+1}, S^{p}\left(t_{n+1}\right)\right) \\
& +\frac{h^{\alpha}}{\Gamma(\alpha+2)} \sum_{j=0}^{n} \Lambda_{j, n+1} \psi_{1}\left(t_{j}, S\left(t_{j}\right)\right), S^{p}\left(t_{n+1}\right) \\
= & \sum_{i=0}^{\lceil\alpha\rceil-1} S_{0}^{(i)} \frac{t_{n+1}^{i+1}}{i !}+\frac{1}{\Gamma(\alpha)} \sum_{k=0}^{n} \Xi_{k, n+1} \psi_{1}\left(t_{k}, S\left(t_{k}\right)\right),
\end{aligned}
$$

where

$$
\Lambda_{j, n+1}= \begin{cases}n^{\alpha+1}-(n-\alpha)(n+1)^{\alpha}, & \text { if } j=0, \\ (n-j+2)^{\alpha+1}+(n-j)^{\alpha+1}-2(n-j+1)^{\alpha+1}, & \text { if } 1 \leq j \leq n, \\ 1 & \text { if } j=n+1,\end{cases}
$$




$$
\Xi_{j, n+1}=\frac{h^{\alpha}}{\alpha}\left((n+1-j)^{\alpha}-(n-j)^{\alpha}\right) .
$$

A complete study of the stability of the method has been produced in [36].

In this part, we have found the numerical simulation of the proposed paradigm via the predictor-corrector PECE method of ABM [35] coded in MATLAB. The behavior of the obtained solution is shown by taking suitable values of the parameters as in [30]: $u_{1}=0.4, \mu=0.3, N=1000, l=$ $0.2, \omega=0.8, u_{2}=0.2, S(0)=900, I(0)=10, R(0)=D(0)=0$, and $\alpha_{1}=\alpha_{2}=\alpha_{3}=\alpha_{4}=\alpha$. In Figure 3, the behavior of $S(t)$ and $I(t)$ populations versus time with different fractional derivative orders is shown. In Figure 4 , the behavior of $R(t$ ) and $D(t)$ populations versus time with different fractional derivative orders is shown. In Figure 5, the dynamics of the populations in $I(t), R(t)$ and $D(t) 3 \mathrm{D}$ space with different fractional derivative orders are shown. In Figure 6, the dynamics of the populations in $S(t), I(t)$ and $D(t) 3 \mathrm{D}$ space with different fractional derivative orders are shown. In Figure 7, the dynamics of the populations in $S(t), I(t)$, and $R(t) 3 \mathrm{D}$ space with different fractional derivative orders are shown. In Figure 8, the dynamics of the populations in $S(t), R(t)$, and $D(t)$ 3D space with different fractional derivative orders are shown. In Figure 9, the Lyapunov exponents concerning the fraction derivative order $\alpha$ are displayed. From Figure 9, we can conclude that the system is unstable in the transient period since LE1 is positive. But as shown LE1 loses its positivity after a short period which indicates that the system settles to its fixed point. In Figure 10, we show the 3D dynamics of the infected population concerning both time and infection rate at fractional derivative order 0.95. In Figure 11, we show the 3D dynamics of the susceptible population concerning both time and infection rate at fractional derivative order 0.95 . There is a quick convergence between the obtained and the exact solution. In complex models, it is well known that a small variation in physical behavior stimulates several new results to understand and analyze nature in a systematic manner.

\section{Fractional Optimal Control (FOCP) for SIRD Paradigm}

In this position, we discuss the optimal control of the fractional-order SIRD paradigm:

$$
\begin{aligned}
D^{\alpha} S(t) & =-u_{1} S I+(\mu-1) N+\omega R+\left(u_{2}-l\right) S, \\
D^{\alpha} I(t) & =u_{1} S I-\left(\omega+u_{2}\right) I-u_{2} S, \\
D^{\alpha} R(t) & =u_{2} I-\omega R, \\
D^{\alpha} D(t) & =\omega I+(l-\mu) N+l S .
\end{aligned}
$$

According to references [37-40], consider the model (70) inside $R^{4}$. The proposed control functions can be for-
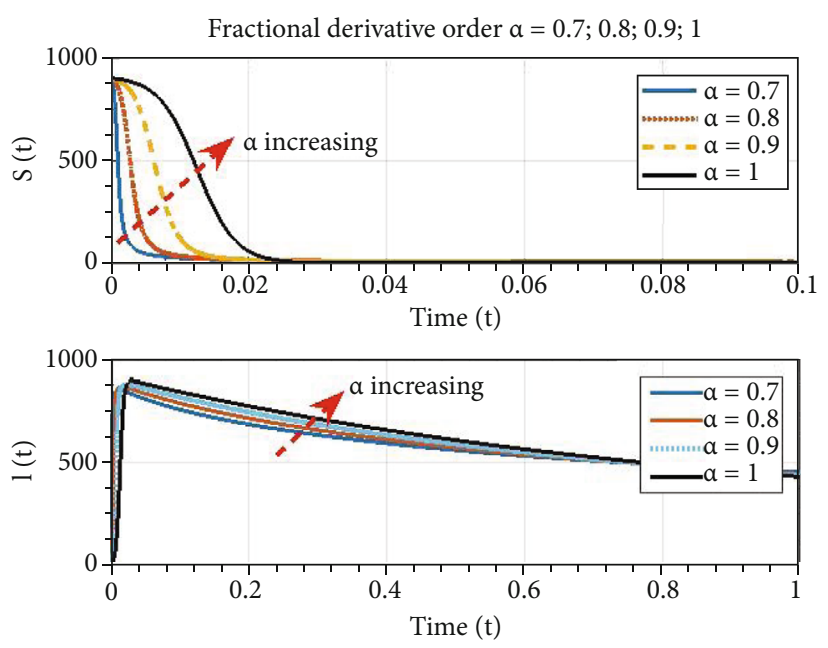

FIgURE 3: The behavior of $S(t)$ and $I(t)$ populations versus time with different fractional derivative orders.

mulated as follows:

$$
\begin{aligned}
\Psi & =\left\{\left(u_{1}(.), u_{2}(.)\right) \in\left(L^{\infty} \times L^{\infty}\right) \mid 0 \leq u_{1}(.), u_{2}(.)\right. \\
& \left.\leq 1, \forall t \in\left[0, T_{f}\right]=[0,1]\right\},
\end{aligned}
$$

and $T_{f}$ refers to the end of the time period, $u_{1}($.$) provides$ the rate of susceptibility per year, and $u_{2}($.$) provides the rate$ of curing from infection population to recovered population per year. $u_{1}($.$) with u_{2}($.$) is the control functions. Here, the$ function space $L^{\infty}$ norm is defined as $\|x\|_{\infty}=\max _{i}\left|x_{i}\right|$. This concept of the $L^{\infty}$ norm considered to be equal to taking the limit as $p \longrightarrow \infty$ of the $L^{p}$ norm.

The following is the definition of the objective function (the control variables are squared)

$$
J\left(u_{1}, u_{2}\right)=\int_{0}^{T_{f}}\left[a I(t)+b u_{1}^{2}(t)+c u_{2}^{2}(t)\right] d t .
$$

Such that $a, b$, and $c$ clarify, respectively, the quantity of infectious, a per year rate of susceptible, and a per year rate of progress from infective to recovered.

Finding the optimal controls $u_{1}($.$) and u_{2}($.$) is the basic$ goal in FOCPs, in order to minimize the predefined objective function:

$$
J\left(u_{1}, u_{2}\right)=\int_{0}^{T_{f}} \psi\left[S, I, R, D, u_{1}, u_{2}, t\right] d t
$$

where $\psi\left[S, I, R, D, u_{1}, u_{2}, t\right]=\left[a I(t)+b u_{1}^{2}(t)+c u_{2}^{2}(t)\right]$, subjected to the constraint

$$
D^{\alpha} S=\eta_{1}, D^{\alpha} I=\eta_{2}, D^{\alpha} R=\eta_{3}, D^{\alpha} D=\eta_{4},
$$

where $\eta_{i}=\eta_{i}\left(S, I, R, D, u_{1}, u_{2}, t\right), i=1,2,3,4$.

The initial conditions are as follows:

$$
S(0)=S_{1}, I(0)=I_{1}, R(0)=R_{1}, D(0)=D_{1} .
$$



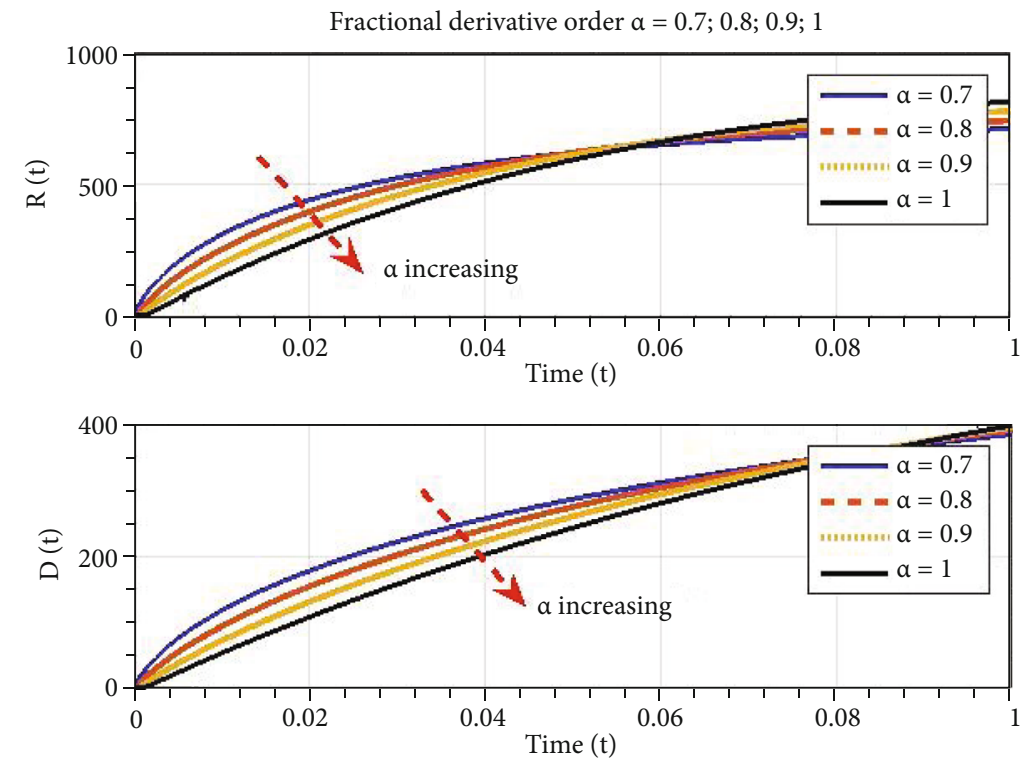

FIgURE 4: The behavior of $R(t)$ and $D(t)$ populations versus time with different fractional derivative orders.

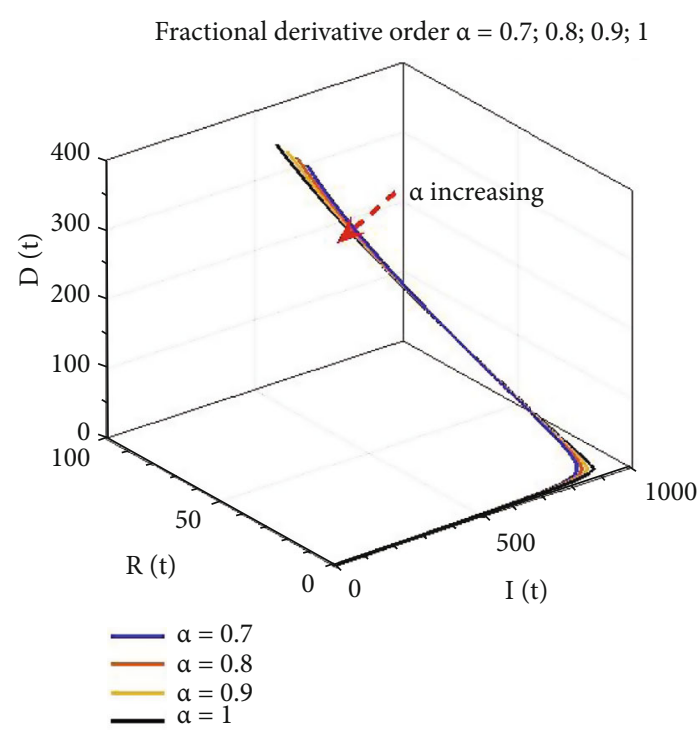

FIGURE 5: The dynamics of the populations in $I(t), R(t)$, and $D(t)$ $3 \mathrm{D}$ space with different fractional derivative orders.

To construct the FOCP, consider the selected objective function defined in [41-44]:

$\stackrel{*}{J}=\int_{0}^{T_{f}}\left[H\left(S, I, R, D, u_{1}, u_{2}, t\right)-\sum_{i=1}^{4} \lambda_{i} \eta_{i}\left(S, I, R, D, u_{1}, u_{2}, t\right)\right] d t$

Such that $i=1,2,3,4$.

In equation (77), (78), we write the Hamiltonian of the cost (objective) function (76) and the control of the fractional-order SIRD paradigm (70):

$$
\begin{aligned}
H\left(S, I, R, D, u_{1}, u_{2}, t\right)= & \psi\left(S, I, R, C, u_{1}, u_{2}, t\right) \\
& +\sum_{i=1}^{4} \lambda_{i} \eta_{i}\left(S, I, R, D, u_{1}, u_{2}, t\right),
\end{aligned}
$$

then

$$
\begin{aligned}
H= & a I+b u_{1}^{2}+c u_{2}^{2}+\lambda_{1}\left[-u_{1} S I+(\mu-1) N\right. \\
& \left.+\omega R+\left(u_{2}-l\right) S\right]+\lambda_{3}\left[u_{2} I-\omega R\right] \\
& +\lambda_{2}\left[u_{1} S I-\left(\omega+u_{2}\right) I-u_{2} S\right] \\
& +\lambda_{4}[\omega I+(l-\mu) N+l S] .
\end{aligned}
$$

From (76) and (78), following the method in [37, 39, 43-45], the FOPC's necessary and sufficient conditions are formulated as

$$
\begin{aligned}
D^{\alpha} \lambda_{1} & =\frac{\partial H}{\partial S}, D^{\alpha} \lambda_{2}=\frac{\partial H}{\partial I}, D^{\alpha} \lambda_{3}=\frac{\partial H}{\partial R}, D^{\alpha} \lambda_{4}=\frac{\partial H}{\partial D}, \\
\frac{\partial H}{\partial u_{k}} & =0, k=1,2 \Rightarrow \frac{\partial H}{\partial u_{1}}=0, \frac{\partial H}{\partial u_{2}}=0, \\
D^{\alpha} S & =\frac{\partial H}{\partial \lambda_{1}}, D^{\alpha} I=\frac{\partial H}{\partial \lambda_{2}}, D^{\alpha} R=\frac{\partial H}{\partial \lambda_{3}}, D^{\alpha} D=\frac{\partial H}{\partial \lambda_{4}},
\end{aligned}
$$

additionally,

$$
\lambda_{i},\left(T_{f}\right)=0
$$

where $\lambda_{i}, i=1,2,3,4$ represent the Lagrange multipliers. In equations (79) and (80), the necessary conditions using FOPC Hamiltonian are represented.

The main result is recorded in Theorem 5 . 


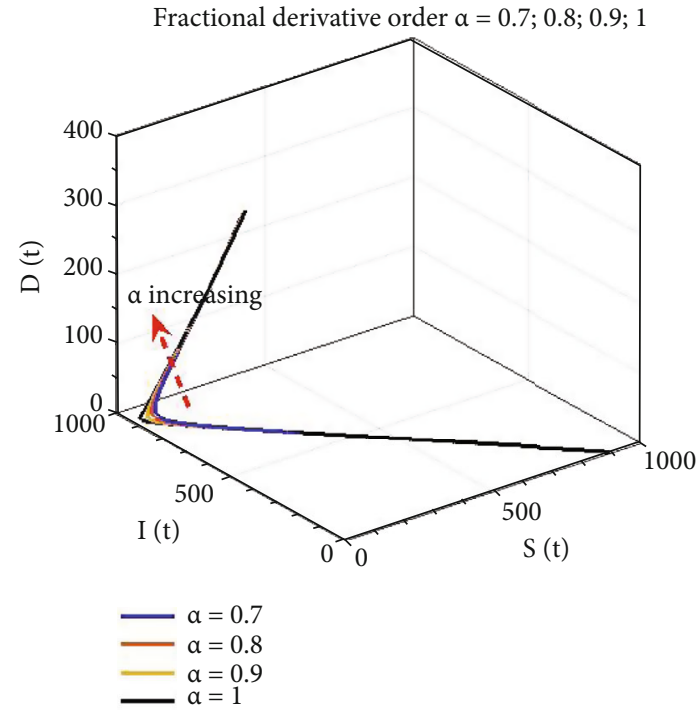

Figure 6: The dynamics of the populations in $S(t), I(t)$, and $D(t)$ $3 \mathrm{D}$ space with different fractional derivative orders.

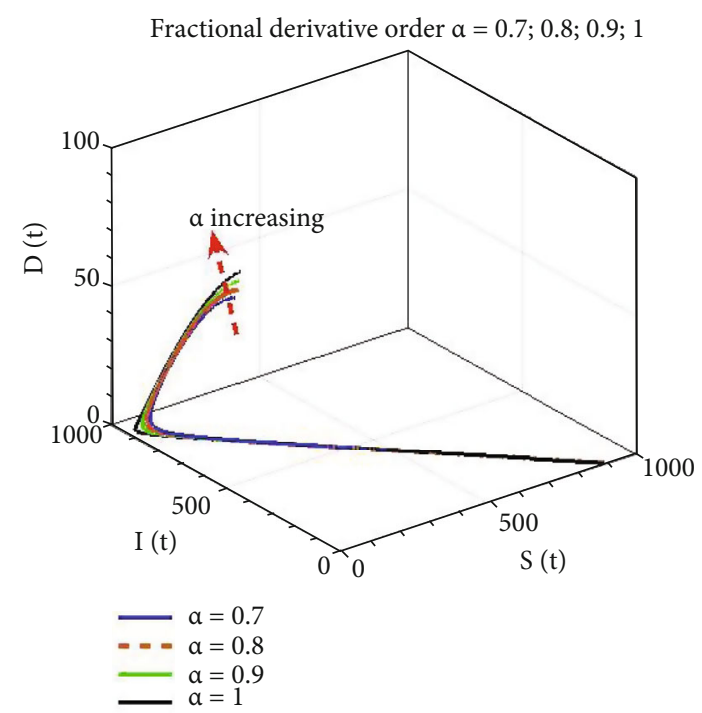

Figure 7: The dynamics of the populations in $S(t), I(t)$, and $R(t)$ $3 \mathrm{D}$ space with different fractional derivative orders.

Theorem 5. If $u_{1}$ and $u_{2}$ are optimal controls related to the controlled states $S^{*}, I^{*}, R^{*}$ and $D^{*}$, then, the adjoint variables $\lambda_{i}^{*}, i=1,2,3,4$ exist that satisfy the following conditions:

(i) Adjoint conditions (costate conditions)

Considering the related conditions represented in the recorded theorem and using equations (82), see [37, 39, 43, 44], we obtain the following four conditions that can be formulated as

$$
\begin{aligned}
D^{\alpha} \lambda_{1}^{*}= & \lambda_{1}^{*}\left[-u_{1} I^{*}+\left(u_{2}-l\right)\right]+\lambda_{2}^{*}\left[u_{1} I^{*}-u_{2}\right]+\lambda_{4}^{*}[l], \\
D^{\alpha} \lambda_{2}^{*}= & a+\lambda_{1}^{*}\left[-u_{1} S^{*}\right]+\lambda_{2}^{*}\left[u_{1} S^{*}-\left(\omega+u_{2}\right)\right] \\
& +\lambda_{3}^{*}\left[u_{2}\right]+\lambda_{4}^{*}[\omega],
\end{aligned}
$$

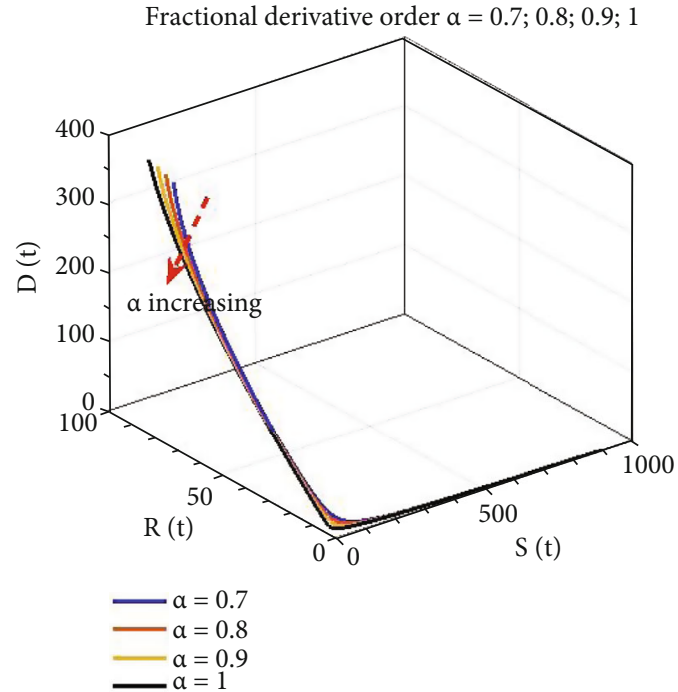

FIgURe 8: The dynamics of the populations in $S(t), R(t)$, and $D(t)$ $3 \mathrm{D}$ space with different fractional derivative orders.

$$
\begin{aligned}
& D^{\alpha} \lambda_{3}^{*}=\lambda_{1}^{*}[\omega]+\lambda_{3}^{*}[-\omega], \\
& D^{\alpha} \lambda_{4}^{*}=0 .
\end{aligned}
$$

(ii) Transversality conditions

$$
\lambda_{i}^{*}\left(T_{f}\right)=0, i=1,2,3,4
$$

(iii) Conditions of optimality

$$
\begin{aligned}
& H\left(S^{*}, I^{*}, R^{*}, D^{*}, u_{1}^{*}, u_{2}^{*}, \lambda_{i}\right) \\
& \quad=\min _{0 \leq u_{1}^{*}, u_{2}^{*} \leq 1} H^{*}\left(S^{*}, I^{*}, R^{*}, D^{*}, u_{1}^{*}, u_{2}^{*}, \lambda_{i}\right),
\end{aligned}
$$

moreover, by applying equations (80), the control functions $u_{1}^{*}, u_{2}^{*}$ are given as follows:

$\frac{\partial H}{\partial u_{1}}=0 \Rightarrow u_{1}=\frac{\left[\lambda_{1}^{*}-\lambda_{2}^{*}\right] S^{*} I^{*}}{2 b}$

$\frac{\partial H}{\partial u_{2}}=0 \Rightarrow u_{2}=\frac{\left[\lambda_{2}^{*}-\lambda_{3}^{*}\right] I^{*}+\left[\lambda_{2}^{*}-\lambda_{1}^{*}\right] S^{*}}{2 c}$,

$u_{1}^{*}=\min \left\{1, \max \left\{0, \frac{\left[\lambda_{1}^{*}-\lambda_{2}^{*}\right] S^{*} I^{*}}{2 b}\right\}\right\}$

$v_{2}^{*}=\min \left\{1, \max \left\{0, \frac{\left[\lambda_{2}^{*}-\lambda_{3}^{*}\right] I^{*}+\left[\lambda_{2}^{*}-\lambda_{1}^{*}\right] S^{*}}{2 c}\right\}\right\}$

Proof. The system of costate conditions (83)-(86) is produced from equation (81) such that the Hamiltonian $H^{*}$ is presented as 

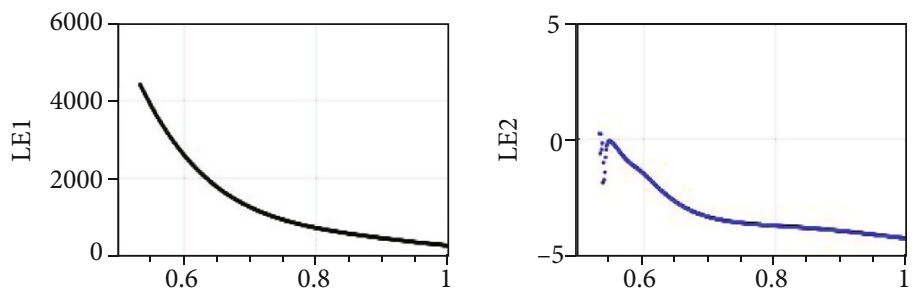

Fractional order : $\alpha$

Fractional order : $\alpha$
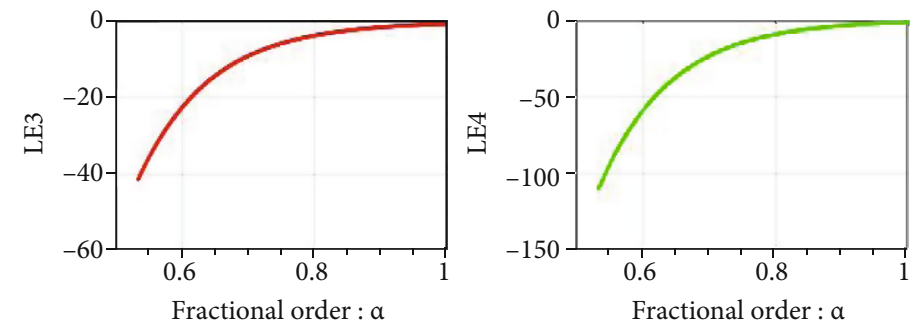

FIgURE 9: The exponents of Lyapunov as a function of the fractional derivative order $\alpha$.

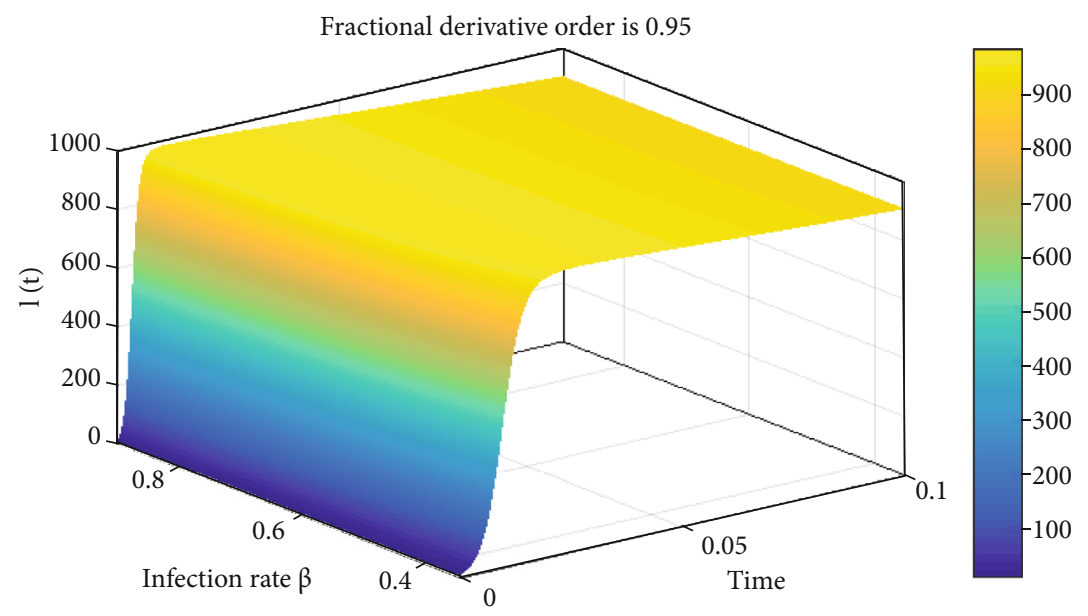

Figure 10: 3D dynamics of the infected population versus both time and infection rate at fractional derivative order 0.95 .

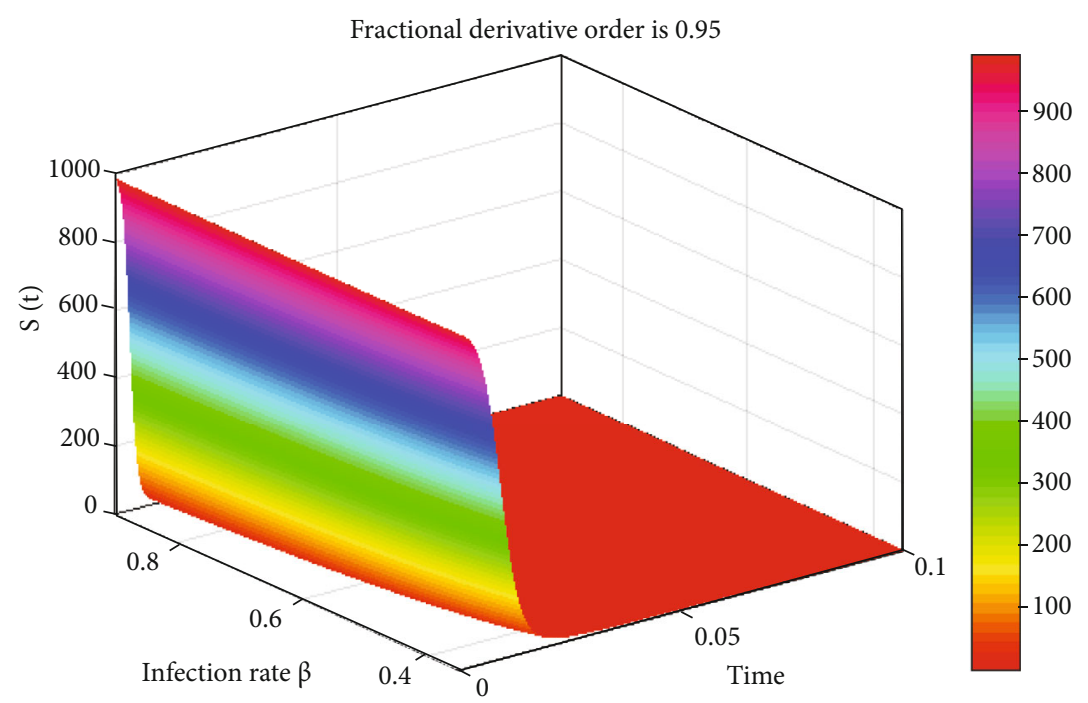

FIgURE 11: 3D dynamics of the susceptible population versus both time and infection rate at fractional derivative order 0.95 . 


$$
\begin{aligned}
H^{*}= & a I^{*}+b u_{1}^{* 2}+c u_{2}^{* 2}+\lambda_{1}^{*} D^{\alpha} S^{*} \\
& +\lambda_{2}^{*} D^{\alpha} I^{*}+\lambda_{3}^{*} D^{\alpha} R^{*}+\lambda_{4}^{*} D^{\alpha} D^{*} .
\end{aligned}
$$

Furthermore, the conditions in equation (82) are guaranteed, and the optimal control given by equations (91)-(92) can be produced via equation (80).

Substituting $v_{i}^{*}, i=1,2$ in (70), the state system is formulated as follows:

$$
\begin{aligned}
D^{\alpha} S^{*}(t) & =-u_{1}^{*} S^{*} I^{*}+(\mu-1) N+\omega R^{*}+\left(u_{2}^{*}-l\right) S^{*}, \\
D^{\alpha} I^{*}(t) & =u_{1}^{*} S^{*} I^{*}-\left(\omega+u_{2}^{*}\right) I^{*}-u_{2}^{*} S^{*}, \\
D^{\alpha} R^{*}(t) & =u_{2}^{*} I^{*}-\omega R^{*} \\
D^{\alpha} D^{*}(t) & =\omega I^{*}+(l-\mu) N+l S^{*} .
\end{aligned}
$$

For more details about FOC, see [37, 39, 40, 44].

The existence of the control system (83)-(86) is to be proved here, the same can be found in [37-43] as follows:

Let

$$
\begin{aligned}
g_{1}\left(\lambda_{1}^{*}, \lambda_{2}^{*}, \lambda_{3}^{*}, \lambda_{4}^{*}\right)= & \lambda_{1}^{*}\left[-u_{1}^{*} I^{*}+\left(u_{2}^{*}-l\right)\right] \\
& +\lambda_{2}^{*}\left[u_{1}^{*} I^{*}-u_{2}^{*}\right]+\lambda_{4}^{*}[l], \\
g_{2}\left(\lambda_{1}^{*}, \lambda_{2}^{*}, \lambda_{3}^{*}, \lambda_{4}^{*}\right)= & a+\lambda_{1}^{*}\left[-u_{1}^{*} S^{*}\right]+\lambda_{2}^{*}\left[u_{1}^{*} S^{*}-\left(\omega+u_{2}^{*}\right)\right] \\
& +\lambda_{3}^{*}\left[u_{2}^{*}\right]+\lambda_{4}^{*}[\omega],
\end{aligned}
$$

$$
g_{3}\left(\lambda_{1}^{*}, \lambda_{2}^{*}, \lambda_{3}^{*}, \lambda_{4}^{*}\right)=\lambda_{1}^{*}[\omega]+\lambda_{3}^{*}[-\omega], g_{4}\left(\lambda_{1}^{*}, \lambda_{2}^{*}, \lambda_{3}^{*}, \lambda_{4}^{*}\right)=0 \text {. }
$$

Let $\Omega=\left\{\lambda_{r}^{*} \in R:\left|\lambda_{r}^{*}\right| \leq k, r=1,2,3,4, t \in[0, T]\right\}$.

We have, at $\Omega: \partial g_{1} / \partial \lambda_{1}^{*}=-u_{1}^{*} I^{*}+\left(u_{2}^{*}-l\right), \partial g_{1} / \partial \lambda_{2}^{*}=$ $u_{1}^{*} I^{*}-u_{2}^{*}, \partial g_{1} / \partial \lambda_{3}^{*}=0, \partial g_{1} / \partial \lambda_{4}^{*}=l, \partial g_{2} / \partial \lambda_{1}^{*}=-u_{1}^{*} S^{*}, \partial g_{2} /$ $\partial \lambda_{2}^{*}=u_{1}^{*} S^{*}-\left(\omega+u_{2}^{*}\right), \partial g_{2} / \partial \lambda_{3}^{*}=u_{2}^{*}, \partial g_{2} / \partial \lambda_{4}^{*}=\omega$,

$$
\begin{aligned}
\frac{\partial g_{3}}{\partial \lambda_{1}^{*}} & =\omega, \frac{\partial g_{3}}{\partial \lambda_{2}^{*}}=0, \frac{\partial g_{3}}{\partial \lambda_{3}^{*}}=-\omega, \frac{\partial g_{3}}{\partial \lambda_{4}^{*}}=0 \\
\frac{\partial g_{4}}{\partial \lambda_{1}^{*}} & =\frac{\partial g_{4}}{\partial \lambda_{2}^{*}}=\frac{\partial g_{4}}{\partial \lambda_{3}^{*}}=\frac{\partial g_{4}}{\partial \lambda_{4}^{*}}=0 \\
\left|\frac{\partial g_{1}}{\partial \lambda_{3}^{*}}\right| & \leq k_{1} \Rightarrow g_{1}=g_{13},\left|\frac{\partial g_{1}}{\partial \lambda_{4}^{*}}\right| \leq g_{1} \Rightarrow g_{1} \\
& =k_{13},|| \frac{\partial g_{1}}{\partial \lambda_{1}^{*}}\left|\leq k_{11}, \frac{\partial g_{1}}{\partial \lambda_{2}^{*}}\right| \leq k_{12}, \\
\left|\frac{\partial g_{2}}{\partial \lambda_{1}^{*}}\right| & \leq k_{21}, \cdots|| \frac{\partial g_{2}}{\partial \lambda_{3}^{*}}\left|\leq k_{23},\right| \frac{\partial g_{2}}{\partial \lambda_{4}^{*}}\left|\leq k_{24}, \frac{\partial g_{2}}{\partial \lambda_{2}^{*}}\right| \leq k_{22}, \cdots \\
\left|\frac{\partial g_{3}}{\partial \lambda_{1}^{*}}\right| & \leq k_{2},\left|\frac{\partial g_{3}}{\partial \lambda_{2}^{*}}\right| \leq k_{2} \Rightarrow g_{3}=k_{31},\left|\frac{\partial g_{3}}{\partial \lambda_{3}^{*}}\right| \leq k_{33},\left|\frac{\partial g_{3}}{\partial \lambda_{4}^{*}}\right| \leq k_{34}
\end{aligned}
$$

$\left|\frac{\partial g_{4}}{\partial \lambda_{1}^{*}}\right| \leq k_{41},|| \frac{\partial g_{4}}{\partial \lambda_{3}^{*}}\left|\leq k_{43},\right| \frac{\partial g_{4}}{\partial \lambda_{4}^{*}}\left|\leq k_{44}, \frac{\partial g_{4}}{\partial \lambda_{2}^{*}}\right| \leq k_{42}$,

where $k_{1}, k_{2}, k_{3}, k_{4}, k_{5} k_{22}, k_{23}, k_{24}, k_{2}, k_{31}, k_{33}, k_{34}, k_{41}, k_{42}, k_{43}$ , and $k_{44}$ are constants greater than zero. Hence, each of the four functions $f_{1}, f_{2}$, and $f_{4}$ agrees with the Lipschitz $z$ conditions. Then, each of the four kernels $f_{1}, f_{2}$ is continuous respecting the four features, then. See for instance [37-43] for more information about the existence and uniqueness.

\section{Conclusion}

In this work, we have provided the approximate solution constructions for the fractional-order LHF model. In addition, the numerical simulations of the fractional-order LHF model have been experimented with many arbitrary orders. Also, an optimal control case has been investigated. The outcomes acquired by utilizing Laplace transform combined with the ADM are very compatible with results existing in the previous research. Further, the applied method for finding the numerical solution of the proposed model is introduced without considering any perturbation, discretization, or transformations. The current examination enlightens the considered nonlinear manner that rely upon the time history and the time moment by applying the idea of fractional order differentiation. Moreover, the obtained results show that Laplace transform combined with the ADM is amazingly deliberate, more powerful, and exceptionally precise, and which can be applied to break down the different classes of nonlinear marvels that emerge in science and innovation. For future work, we suggest generalizing the studied LHF model to show the effect of the infection from other sources as animals, men, and children.

\section{Data Availability}

The data used to support the findings of this study are available from the corresponding author on request.

\section{Conflicts of Interest}

The authors declare no conflict of interest.

\section{Authors' Contributions}

Conceptualization was done by M.H.; methodology was done by M.H, A.E., and A.M.; software was done by M.H, A.E., and A.M.; validation was done by M.H, A.E., S.U., A.G., and A.M.; formal analysis was done by M.H, A.E., S.U., A.G., and A.M.; investigation was done by M.H, A.E., S.U., A.G., and A.M.; resources was done by M.H, A.E., S.U., A.G., and A.M.; data curation was done by M.H, A.E., S.U., A.G., and A.M.; writing-original draft preparation was done by M.H. and A.E.; writing-review and editing was done by M.H. and A.E.; visualization was done by M.H. and A.E.; supervision was done by M.H.; project administration was done by M.H.; funding acquisition was 
done by A.G. and S.U. All authors have read and agreed to the published version of the manuscript.

\section{Acknowledgments}

The authors acknowledge the support and funding of Research Center for Advanced Material Science (RCAMS) at King Khalid University through Grant No. RCAMS/ KKU/008-21.

\section{References}

[1] J. M. van Seventer and N. S. Hochberg, "Principles of infectious diseases: transmission, diagnosis, prevention, and control," Public Health, pp. 22-39, 2017.

[2] O. Ogbu, E. Ajuluchukwu, and C. J. Uneke, "Lassa fever in West African sub-region: an overview," Journal of Vector Borne Diseases, vol. 44, no. 1, pp. 1-11, 2007.

[3] J. D. Frame, J. M. Baldwin Jr., D. J. Gocke, and J. M. Troup, "Lassa fever, a new virus disease of man from West Africa," The American Journal of Tropical Medicine and Hygiene, vol. 19, no. 4, pp. 670-676, 1970.

[4] I. Faith, O. A. A. SM, O. Ifeanyi et al., "An assessment of onsetto-intervention time and outcome of Lassa fever during an outbreak in Edo State, Nigeria," International Journal of Prevention and Treatment, vol. 7, no. 1, pp. 1-5, 2018.

[5] H. U. Ekechi, C. Ibeneme, B. Ogunniyi et al., "Factors associated with a confirmed Lassa fever outbreak in Eguare community of Esan West, Edo State, Nigeria: January-March, 2019," Journal of Interventional Epidemiology and Public Health, vol. 3, no. 1, 2020.

[6] J. A. Tenreiro Machado, M. F. Silva, R. S. Barbosa et al., "Some applications of fractional calculus in engineering," Mathematical Problems in Engineering, vol. 2010, Article ID 639801, 34 pages, 2010.

[7] D. Baleanu, Z. B. Guvenc, and J. A. T. Machado, New Trends in Nanotechnology and Fractional Calculus Applications, Springer Dordrecht Heidelberg, London, New York, 2010.

[8] H. Bulut, T. A. Sulaiman, H. M. Baskonus, H. Rezazadeh, M. Eslami, and M. Mirzazadeh, "Optical solitons and other solutions to the conformable space-time fractional FokasLenells equation," Optik, vol. 172, pp. 20-27, 2018.

[9] P. Veeresha, D. G. Prakasha, and H. M. Baskonus, "Solving smoking epidemic model of fractional order using a modified homotopy analysis transform method," The Mathematical Scientist, vol. 13, no. 2, pp. 115-128, 2019.

[10] D. Baleanu, G. C. Wu, and S. D. Zeng, "Chaos analysis and asymptotic stability of generalized Caputo fractional differential equations," Chaos, Solitons \& Fractals, vol. 102, pp. 99105, 2017.

[11] R. S. Barbosa, J. A. T. Machado, and I. M. Ferreira, "PID controller tuning using fractional calculus concepts," Fractional Calculus \& Applied Analysis, vol. 7, no. 2, pp. 119-134, 2004.

[12] M. F. Silva, J. A. T. Machado, and A. M. Lopes, "Position/force control of a walking robot," Machine Intelligence and Robot Control, vol. 5, pp. 33-44, 2003.

[13] M. F. Silva, J. A. T. Machado, and A. M. Lopes, "Comparison of fractional and integer order control of an hexapod robot," in Proceedings of International Design Engineering Technical Conferences and Computers and Information in Engineering Conference, vol. 5 of 19th Biennial Conference on Mechani- cal Vibration and Noise, pp. 667-676, Chicago, Ill, USA, 2003.

[14] R. S. Barbosa, J. A. T. Machado, and I. M. Ferreira, "Tuning of PID controllers based on Bode's ideal transfer function," Nonlinear Dynamics, vol. 38, no. 1-4, pp. 305-321, 2004.

[15] F. Duarte and J. A. T. Machado, "Chaotic phenomena and fractional-order dynamics in the trajectory control of redundant manipulators," Nonlinear Dynamics, vol. 29, no. 1/4, pp. 315-342, 2002.

[16] M. F. Silva and J. A. T. Machado, "Fractional order PD $\alpha$ joint control of legged robots," Journal of Vibration and Control, vol. 12, no. 12, pp. 1483-1501, 2006.

[17] J. A. T. Machado, I. S. Jesus, J. B. Cunha, and J. K. Tar, "Fractional dynamics and control of distributed parameter systems," Intelligent Systems at the Service of Mankind, vol. 2, pp. 295-305, 2006.

[18] J. A. T. Machado, "Discrete-time fractional-order controllers," Fractional Calculus \& Applied Analysis, vol. 4, no. 1, pp. 47-66, 2001.

[19] A. I. El-Mesady, Y. S. Hamed, and A. M. Alsharif, "Jafari transformation for solving a system of ordinary differential equations with medical application," Fractal and Fractional, vol. 5, no. 3, p. 130, 2021.

[20] N. H. Sweilam, S. M. al-Mekhlafi, A. O. Albalawi, and J. A. Tenreiro Machado, "Optimal control of variable-order fractional model for delay cancer treatments," Applied Mathematical Modelling, vol. 89, pp. 1557-1574, 2021.

[21] A. Atangana, "A novel model for the Lassa hemorrhagic fever: deathly disease for pregnant women," Neural Computing and Applications, vol. 26, no. 8, pp. 1895-1903, 2015.

[22] M. Goyal, H. M. Baskonus, and A. Prakash, “An efficient technique for a time fractional model of Lassa hemorrhagic fever spreading in pregnant women," The European Physical Journal Plus, vol. 134, no. 10, pp. 1-10, 2019.

[23] M. Abdelhakem, T. Alaa-Eldeen, D. Baleanu, M. G. Alshehri, and M. El-Kady, "Approximating real-life BVPs via Chebyshev polynomials' first derivative pseudo-Galerkin method," Fractal and Fractional, vol. 5, no. 4, p. 165, 2021.

[24] M. Abdelhakem and Y. H. Youssri, "Two spectral Legendre's derivative algorithms for Lane-Emden, Bratu equations, and singular perturbed problems," Applied Numerical Mathematics, vol. 169, pp. 243-255, 2021.

[25] Y. H. Youssri, W. M. Abd-Elhameed, and M. Abdelhakem, "A robust spectral treatment of a class of initial value problems using modified Chebyshev polynomials," Mathematical Methods in the Applied Sciences, vol. 44, no. 11, pp. 92249236, 2021.

[26] M. Abdelhakem, M. Biomy, A. S. Kandil, and D. Baleanu, "A numerical method based on Legendre differentiation matrices for higher order ODEs," Information Sciences Letters, vol. 9, no. 3, pp. 175-180, 2020.

[27] M. Abdelhakem, D. Abdelhamied, A. Mg, and E.-K. Ma, "Shifted Legendre fractional pseudospectral differentiation matrices for solving fractional differential problems," Fractals, no. article 2240038, 2021.

[28] M. Abdelhakem, D. Mahmoud, D. Baleanu, and M. el-kady, "Shifted ultraspherical pseudo-Galerkin method for approximating the solutions of some types of ordinary fractional problems," Advances in Difference Equations, vol. 2021, no. 1, 18 pages, 2021. 
[29] M. Abdelhakem, H. Moussa, D. Baleanu, and M. El-Kady, "Shifted Chebyshev schemes for solving fractional optimal control problems," Journal of Vibration and Control, vol. 25, no. 15, pp. 2143-2150, 2019.

[30] W. Gao, P. Veeresha, D. G. Prakasha, H. M. Baskonus, and G. Yel, "New approach for the model describing the deathly disease in pregnant women using Mittag-Leffler function," Chaos, Solitons \& Fractals, vol. 134, article 109696, 2020.

[31] S. Jain and A. Atangana, "Analysis of Lassa hemorrhagic fever model with non-local and non-singular fractional derivatives," International Journal of Biomathematics, vol. 11, no. 8, p. 1850100, 2018.

[32] A. Yusuf, T. Sulaiman, and P. Kumar, A New Study of Lassa Hemorrhagic Fever Model via Caputo-Fabrizio Derivative, Authorea, 2020.

[33] Y. A. Amer, A. M. S. Mahdy, T. T. Shwayaa, and E. S. M. Youssef, "Laplace transform method for solving nonlinear biochemical reaction model and nonlinear Emden-Fowler system," Journal of Engineering and Applied Sciences, vol. 13, no. 17, 2018.

[34] P. van den Driessche and J. Watmough, "Reproduction numbers and sub-threshold endemic equilibria for compartmental models of disease transmission," Mathematical Biosciences, vol. 180, no. 1-2, pp. 29-48, 2002.

[35] K. Diethelm and A. D. Freed, "The FracPECE subroutine for the numerical solution of differential equations of fractional order," in Research and Scientific Computing 1998, S. Heinzel and T. Plesser, Eds., pp. 57-71, Society for scientific data processing, Göttingen, 1999.

[36] R. Garrappa, "On linear stability of predictor-corrector algorithms for fractional differential equations," International Journal of Computer Mathematics, vol. 87, no. 10, pp. 22812290, 2010.

[37] A. M. S. Mahdy, K. A. Gepreel, K. Lotfy, and A. A. El-Bary, “A numerical method for solving the Rubella ailment disease model," International Journal of Modern Physics C, vol. 32, no. 7, pp. 1-15, 2021.

[38] K. A. Gepreel, M. Higazy, and A. M. S. Mahdy, "Optimal control, signal flow graph, and system electronic circuit realization for nonlinear Anopheles mosquito model," International Journal of Modern Physics C, vol. 31, no. 9, pp. 1-18, 2020.

[39] A. M. S. Mahdy, M. S. Mohamed, K. Lotfy, M. Alhazmi, A. A. El-Bary, and M. H. Raddadi, "Numerical solution and dynamical behaviors for solving fractional nonlinear rubella ailment disease model," Results in Physics, vol. 39, pp. 1-10, 2018.

[40] N. H. Sweilam, S. M. Al-Mekhlafi, and D. Baleanu, "Optimal control for a fractional tuberculosis infection model including the impact of diabetes and resistant strains," Journal of Advanced Research, vol. 24, pp. 125-137, 2019.

[41] A. Jajarmi, B. Ghanbari, and D. Baleanu, "A new and efficient numerical method for the fractional modeling and optimal control of diabetes and tuberculosis co-existence," Chaos, vol. 29, no. 9, article 093111, 2019.

[42] M. Higazy, F. M. Allehiany, and E. E. Mahmoud, "Numerical study of fractional order COVID-19 pandemic transmission model in context of ABO blood group," Results in Physics, vol. 22, article 103852, 2021.

[43] G. Yel and H. M. Baskonus, "Solitons in conformable timefractional Wu-Zhang system arising in coastal design," Pramana, vol. 93, no. 4, pp. 1-10, 2019.
[44] W. Gao, H. F. Ismael, A. M. Husien, H. Bulut, and H. M. Baskonus, "Optical soliton solutions of the cubic-quartic nonlinear Schrödinger and resonant nonlinear Schrödinger equation with the parabolic law," Applied Sciences, vol. 10, no. 1, p. 219, 2020.

[45] A. M. S. Mahdy, M. Higazy, and M. S. Mohamed, "Optimal and memristor-based control of a nonlinear fractional tumor-immune model," Materials \& Continua, vol. 67, no. 3, pp. 3463-3486, 2021. 